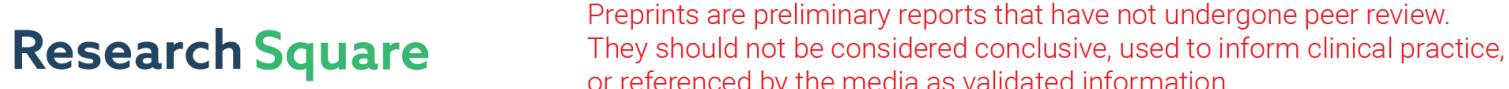 or referenced by the media as validated information. \\ Transfer Method of Geometric Tolerance Items Based on Assembly Joints
}

\section{Ning MA ( 1768567565@qq.com )}

University of Jinan https://orcid.org/0000-0003-4142-3280

\section{Bo YANG \\ University of Jinan \\ Jinping LI \\ University of Jinan \\ Yanchao LIU \\ University of Jinan \\ Dianxi WANG \\ University of Jinan \\ Changqing GAO \\ University of Jinan}

\section{Research Article}

Keywords: ometric tolerance items, Assembly joints, Transfer path, Functional requirements, Structural constraints

Posted Date: February 12th, 2021

DOI: https://doi.org/10.21203/rs.3.rs-207551/v1

License: (c) (i) This work is licensed under a Creative Commons Attribution 4.0 International License. Read Full License

Version of Record: A version of this preprint was published at The International Journal of Advanced Manufacturing Technology on August 11th, 2021. See the published version at https://doi.org/10.1007/s00170-021-07598-y. 


\title{
Transfer Method of Geometric Tolerance Items Based on Assembly Joints
}

\author{
Ning Ma ${ }^{1}$ Bo Yang ${ }^{1 *}$ Jinping Li $^{1}$ Yanchao Liu ${ }^{1}$ Dianxi Wang ${ }^{1}$ Changqing Gao ${ }^{1}$ \\ 1. School of Mechanical Engineering, University of Jinan, Jinan 250022 \\ 1*E-mail: me_yangb@ujn.edu.cn
}

\begin{abstract}
In order to reduce the uncertainty in the selection of geometric tolerance items, a qualitative method for top-down transfer of geometric tolerance items based on assembly joints is proposed. According to the structural characteristics, the assembly joints are divided into meta-assembly joints and composite assembly joints, and the priority rules for assembly joints are proposed. The transfer path of part-level geometric tolerance items is established according to the functional requirements and structural constraints among parts. On this basis, by adding information about the composition and constraint types of assembly joints between parts and the positioning constraint relationship of the general structure surface in the part, the transfer path of part-level geometric tolerance items is extended to the surface-level transfer path. The structural transformation rules for functional requirements based on structural constraints, the tolerance item generation specifications and datum transfer specifications are established. And based on the above specifications, the mapping relationship between functional requirements, structural constraints and geometric tolerance items is defined. The synchronous transmission of geometric tolerance items along with the product design process are realized which provides an effective analysis tool for the top-down design of geometric tolerance items. Finally, the effectiveness of the method is verified by taking the transmission parts and connection parts in the crankshaft-piston mechanism as an example.
\end{abstract}

Key words: Geometric tolerance items; Assembly joints; Transfer path; Functional requirements; Structural constraints

\section{Introduction}

With the continuous development of high-precision machinery and equipment, geometric tolerance design has become a key link that must be considered in the product design stage. In traditional tolerance design, the selection of geometric tolerance items often depends on the designer's experience, which has great uncertainty. It also affects the quality characteristics of parts such as matching and service life. How to analyze the functional characteristics and structural attributes of the product itself according to the design requirements, provide a qualitative method for geometric tolerance design, and realize the top-down transfer and generation of geometric tolerance items is an important problem in this field.

At present, many scholars at home and abroad have explored related problems, and the methods proposed are roughly divided into the following three types: (1) Positioning rule method. This method is mainly based on the positioning features such as the assembly and positioning information of the geometric element. By the systematic analysis of positioning features, the tolerance model and the specifications of the geometric tolerance design are constructed, and the geometric tolerance items are generated. For example, by analyzing the functional requirements corresponding to the joints between components, a method of generating functional specifications and functional tolerances based on positioning features was proposed by Anselmetti [1] ; Through studying the expression and decomposition methods of geometric functional requirements, and by using improved assembly directed graphs, a functional tolerance design method based on the evolution from geometric functional requirements to geometric specifications was presented by Yang et al. [2]; By classifying and sorting the positioning features, and using the positioning map to describe the assembly positioning of the parts, the tolerance specification method of the positioning features in the datum 
reference system was given by Gong et al.[3], which is helpful in the automatic design of parts tolerance; Zhang et al. [4] proposed a method of generating tolerance specifications and tolerance domain types based on assembly positioning, which used polychromatic set theory to describe the reasoning process of geometric tolerance items. (2) Theoretical rule method. In this method, a series of theoretical models are introduced to normalize the inference experience of geometric tolerance items selection, and the tolerance items are generated based on this. Armillotta [5] proposed a method of generating tolerance specifications based on product data. This method realized the reasoning of geometric tolerance items based on assembly requirements; Jiang et al. [6] presented a geometric tolerance information inference based on polychromatic set theory and its normative verification method, which improves the accuracy and efficiency of geometric tolerance design; By using the crawler graph to identify the position of the functional surface, a 3D manufacturing tolerance synthesis algorithm based on technology and topology-related surface rules was adopted by Jaballi [7]; In order to analyze error and its stack-up of mechanical product effectively, Zhang et al. [8] presented an integrated modeling method of unified tolerance representation based on Key Features (KFs) and Graph Theory in order to deal with these types of tolerances simultaneously. The number of recommended assembly tolerance types generated can be further reduced on the basis of this; Start from the basic spatial relationship between geometric elements, the description logic of geometric tolerance, the judgment algorithm and the automatic generation algorithm of the tolerance type were proposed by Qin et al. [9]; To realize the automatic generation of the geometric tolerance area in the CAD system, a method based on description logic was proposed by the research group in paper 10 and paper 11; Zhong et al. [12,13] used description logic to construct a meta-model of assembly tolerance items, and automatically generated tolerance items based on the ontology. This method reduces the uncertainty of geometric tolerance items through the rules of description logic; Zhao et al. [14] proposed a novel method for computer-aided tolerance specification to evaluate static factors and dynamic factors. Those factors are assessed by the rule-based algorithm and axiomatic design algorithm. (3) Case-based inference. This method is based on the existing successful cases for analogy and learning, so as to form a generation method of geometric tolerance items. For example, Sarigecili et al. [15] mapped the geometric dimensions and tolerance information of the product from STEP to the OWL-based model, and used the ontology-based model to explain tolerance analysis; Goetza S et al. [16] presented a novel ontology-based approach combining knowledge from the product design and tolerancing domains to enable an automated tolerance specification of product concepts; This method realized the automatic generation of geometric tolerance items by analogy.; In paper 17, the semantic web rule language is used to define and code the tolerance analysis specifications extracted from the ontology, based on which the automatic reasoning of semantic information is realized.

The above method has largely solved the uncertainty in the geometric tolerance design process, and provided a more reliable model and related inference rules for the selection of geometric tolerance items. However, there are still some limitations. Among them, the positioning rule method is mostly based on the functional requirements of the assembly joints between the parts to select geometric items, the structural correlation between the general structure surfaces in the parts was ignored; The theoretical rules have problems with the accuracy and adaptability of the rules; Case-based inference may be subject to variation in the analogy process, resulting in errors in recommended tolerance items.

To solve the above problems, a top-down transfer and design method of geometric tolerance items based on assembly joints was proposed based on the initial functional requirements. The assembly joint was taken as the basic unit of the geometric tolerance item transmission. Through the structural transformation of functional requirements, the generating specification of geometric tolerance items and the transfer specification of datum based on structural correlation 
were established. And the mapping relationship among functional requirements, structural correlation and geometric tolerance items were established. By analyzing the function and structure of the product, the transfer path of part-level and surface-level consistent with the design process is constructed, and the top-down transfer process of geometric tolerance items is realized.

2 Basic unit of the geometric tolerance item transmission

\subsection{Definition of assembly joints}

In the design of the product structure, the assembly relationship is realized through several pairs of matching constraints between surfaces. The basic assembly unit composed of mating surface and matching constraint is mating surface pairs. In actual assembly, the assembly constraint of parts is often composed of multiple, mating surface pairs.

The assembly joint refers to the set of mating surface pairs that cooperate between two parts. As shown in Fig.1, the mating surfaces $F_{1,1}$ and $F_{2,1}$ are attached to form mating surface pair $\mathrm{p}_{11,21}, \mathrm{~F}_{1,2}$ and $\mathrm{F}_{2,2}$ are attached to form $\mathrm{p}_{12,22}$; Then mating surface pairs $\mathrm{p}_{11,21}, \mathrm{p}_{12}, 22$ constitute the assembly joint between the parts $\mathrm{P}_{1}$ and $\mathrm{P}_{2}$.

The assembly joint is the basic carrier of assembly connection and geometric tolerance item transmission, which expresses the spatial shape and positioning relationship and the matching characteristics between matching parts. Complex assembly joints are often composed of simple assembly joints.

Definition 1 meta-assembly joint: Two geometric feature surfaces between the parts have a single spatial constraint due to functional requirements or structural constraints, and the resulting fit surface is called meta-assembly joint.

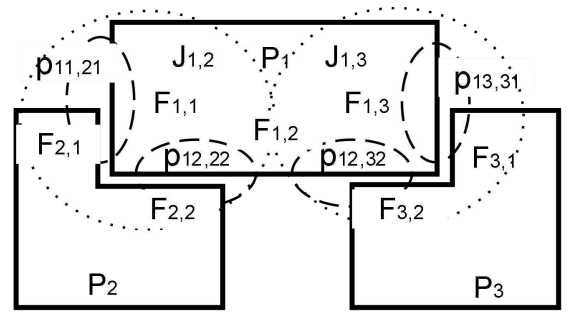

Fig.1 The relationship between assembly joint and mating surface pairs

The type of mating surface and the mating constraint relationship between the mating surfaces are the basic elements of meta-assembly joints. In engineering, common mating surfaces mainly include plane, cylinder, spherical, etc., and the mating constraint relationships between mating surfaces mainly include fit, coaxial and tangent. The meta-assembly joint can be obtained by adding the corresponding mating constraint between the mating surfaces. The common meta-assembly joints are shown in Table 1.

The meta-assembly joint $M J_{i s, j t}$ can be expressed as:

$$
M J_{i s, j t}=F_{i s} \stackrel{C_{i s, j t}}{\longrightarrow} F_{j t}
$$

Where, $F_{i s}$ represents the $s$-th mating surface of the part $P_{i}, F_{j t}$ represents the $t$-th mating surface of the part $P_{j}$ that has a mating constraint with $F_{i s} ; C_{i s, j t}$ represents the matching constraint relationship between the surface $F_{i s}$ and $F_{j t}$, which mainly includes the fit, coaxial, tangent, etc.

Table 1 Common meta-assembly joints

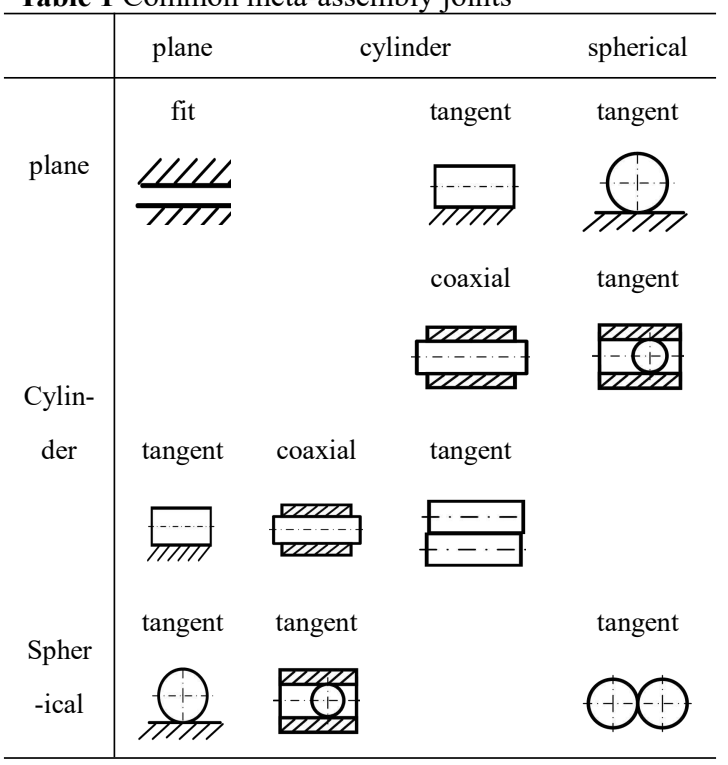

Definition 2 composite assembly joints: The set of multi-component assembly joints with a certain spatial positioning relationship between two parts to ensure the realization of specific functions is called composite assembly joints; Composite assembly joints can be transformed into multiple sets of meta-assembly joints constrained by a certain spatial positioning relation.

The composite assembly joint $J_{i, j}\left(f_{m}\right)$ between the $P_{i}$ and the $P_{j}$ for the completion of a specific function $f_{m}$ can be expressed as: 


$$
\begin{aligned}
& J_{i, j}\left(f_{m}\right)=M J_{i 1, j 1} \stackrel{T_{1,2}}{\longrightarrow} M J_{i 2, j 2} \stackrel{T_{2,3}}{\longrightarrow} \ldots \\
& \stackrel{T_{\mathrm{n}-1, \mathrm{n}}}{\longrightarrow} M J_{i \mathrm{n}, j \mathrm{n}}
\end{aligned}
$$

Where, the constraint $T_{n-1, n}$ is mainly used to describe the direction and positioning constraints relationship between the meta-assembly joints $M J_{i n-1 \text {, }}$ $j n-1$ and $M J_{i n, j n}$, including the direction constraints (parallel, vertical, inclined) and position constraints (fit, coaxial, symmetrical), etc.

The common composite assembly joints are shown in Table 2.

\subsection{Priority of assembly joints}

When there are multiple meta-assembly joints between mating parts, in order to determine the priority of tolerance transfer, the priority of the

\begin{tabular}{|c|c|c|c|}
\hline meta-assembly joint 1 & meta-assembly joint 2 & constraints & $\begin{array}{c}\text { composite assembly } \\
\text { joint }\end{array}$ \\
\hline$\frac{11212}{7777}$ & $\frac{11211}{7777}$ & parallel & \\
\hline$M J_{I}=$ plane $\stackrel{\text { fit }}{\longrightarrow}$ plane & $M J_{2}=$ plane $\stackrel{\text { fit }}{\longrightarrow}$ plane & $\begin{array}{l}\text { vertical } \\
\text { Symmetri- } \\
\quad \text { cal }\end{array}$ & \\
\hline$\frac{11121}{7777}$ & ש200 & vertical & 将 \\
\hline$M J_{I}=$ plane $\stackrel{\text { fit }}{\longrightarrow}$ plane & $M J_{2}=$ cylinder $\stackrel{\text { coaxial }}{\longrightarrow}$ cylinder & & $J=M J_{1} \stackrel{\text { vertical }}{\longrightarrow} M J_{2}$ \\
\hline שבד & שב्य & coaxial & \begin{tabular}{|l|l|} 
\\
\end{tabular} \\
\hline$M J_{1}=$ cylinder $\stackrel{\text { coaxial }}{\longrightarrow}$ cylinder & $M J_{2}=$ cylinder $\stackrel{\text { coaxial }}{\longrightarrow}$ cylinder & & $J=M J_{1} \stackrel{\text { coaxial }}{\longrightarrow} M J_{2}$ \\
\hline שבाम4 & एस्यम & parallel & \\
\hline$M J_{I}=$ cylinder $\stackrel{\text { coaxial }}{\longrightarrow}$ cylinder & $M J_{2}=$ cylinder $\stackrel{\text { coaxial }}{\longrightarrow}$ cylinder & & $J=M J_{1} \stackrel{\text { parallel }}{\longrightarrow} M J_{2}$ \\
\hline
\end{tabular}
Table 2 Common composite assembly joints

As shown in Fig. 2a, there is a meta-assembly joint $\mathrm{MJ}_{11,21}$ formed by large planes fitting and a meta-assembly joint $\mathrm{MJ}_{12,22}$ formed by short cylinders coaxially. The number of constrained degrees of meta-assembly joints should be ordered. The assembly between parts is realized by adding constraints to the mating surfaces. The essence is to restrict the freedom of the parts, so as to realize the positioning and assembly of the parts. Obviously, the more the number of degrees of freedom restricted by the assembly joint, the higher the priority of the assembly joint, and the higher the priority of the transfer path through the joint. And according to the order of limiting the number of degrees of freedom from more to less, the primary assembly joint, secondary assembly joint and third assembly joint are defined in turn. 
there is a meta-assembly joint $\mathrm{MJ}_{11,21}$ formed by small planes fitting and a meta-assembly joint $\mathrm{MJ}_{12,22}$ formed by long cylinders coaxially. The number of constrained degrees of freedom for the small planes is 1 , and the number of constrained degrees of freedom for the long cylinders is 4 ; Therefore, $\mathrm{MJ}_{11,21}$ is the secondary assembly joint and $\mathrm{MJ}_{12,22}$ is the primary assembly joint.

When the inherent attributes (surface type, size, etc.) of the mating surface are consistent, the contact form between the mating surfaces affects the limited degree of freedom. The number of degrees of freedom restricted by plane contact is the largest, followed by the number of degrees of freedom restricted by line contact, and the least number of degrees of freedom restricted by point contact. As shown in Fig. 3, $\mathrm{MJ}_{11,21}$, $\mathrm{MJ}_{12,22}$ are all meta-assembly joints formed by large planes fitting. In Fig. 3a, under the action of gravity, the meta-assembly joint $\mathrm{MJ}_{12,22}$ is approximately plane contact, limiting 3 degrees of freedom. While the joint $\mathrm{MJ}_{11,21}$ is approximately line contact, limiting 2 degrees of freedom. Therefore, the former is the primary assembly joint and the latter is the secondary assembly joint. In Fig. 3b, the screw makes the meta-assembly joint $\mathrm{MJ}_{11,21}$ approximate to plane contact, limiting 3 degrees of freedom; $\mathrm{MJ}_{12,22}$ is approximate to line contact, limiting 2 degrees of freedom. Therefore, $\mathrm{MJ}_{11,21}$ is the primary assembly joint, $\mathrm{MJ}_{12,22}$ is the secondary assembly joint.

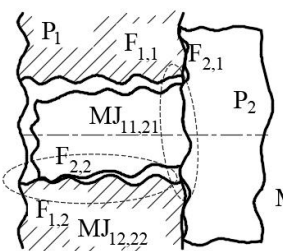

(a)

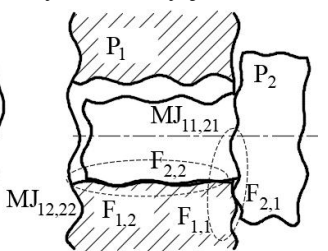

(b)
Fig. 2 Schematic diagram 1 of the priority of assembly joints

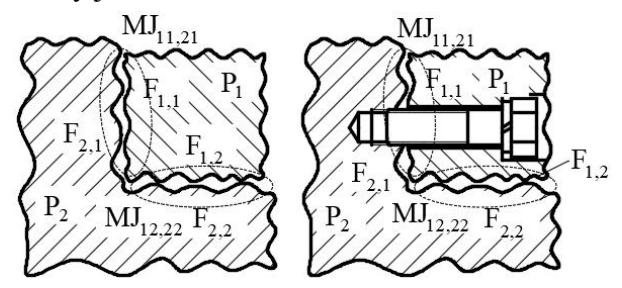

(a)

(b)

Fig. 3 Schematic diagram 2 of the priority of assembly joints

\section{Transfer path of geometric tolerance items}

3.1 Transfer path of part-level geometric tolerance items

The transfer path of geometric tolerance items is first generated based on the correlation between the parts. The transfer path of part-level starts from the part that is initially required to act, and points to the lower part with structural constraints or functional requirements, until the lower part is the last part. According to the correlation between upper and lower parts, the transfer path of part-level can be divided into four basic forms: series path, parallel path, star path and triangle path. On this basis, a mixed path which may be composed of multiple basic paths can be formed.

The series path is shown in Fig. 4a, and the path is $\mathrm{P}_{1} \rightarrow \mathrm{P}_{2} \rightarrow \mathrm{P}_{3}$;

The parallel paths include single-input parallel paths (Fig. 4b) and multi-input parallel paths (Fig. 4c). The star paths (Fig. 4d) refer to multiple unrelated parallel parts that have functional requirements or structural constraints with the same upper part. In the process of tolerance transfer, both parallel paths and star paths have a number of one-way paths with common nodes. In order to simplify the expression of the tolerance transfer process, it is necessary to expand the common nodes to obtain several series paths. For example, the parallel path shown in Fig. $4 \mathrm{~b}$ can be decomposed into two series paths $\mathrm{P}_{1} \rightarrow \mathrm{P}_{2} \rightarrow \mathrm{P}_{4}, \mathrm{P}_{1} \rightarrow \mathrm{P}_{3} \rightarrow \mathrm{P}_{4}$.

In the triangle path (Fig. 4e), there are loops of upper and lower parts. In the construction of the path, different parallel parts can be selected in turn as the last part to obtain several series paths. In Fig. 4e, the triangle path can be decomposed into two series paths $\mathrm{P}_{1} \rightarrow \mathrm{P}_{2} \rightarrow \mathrm{P}_{3} \quad \mathrm{P}_{1} \rightarrow \mathrm{P}_{3} \rightarrow \mathrm{P}_{2}$. Based on the connection characteristics of triangle paths, polygonal paths can be derived.

3.2 Transfer path of surface-level geometric tolerance items

According to the correlation between geometric feature surfaces in the part-level transfer path, the transfer path of part-level can be further expressed as a transfer path of surface-level. It consists of the geometric feature surface, the relationship between 
the surface, and the information about start position and direction. It can be constructed according to the following steps:

(1) Structural transformation of initial functional requirement

The initial design requirement is determined according to the functional requirements, and it can be transformed into a constraint correlation between the two related feature surfaces $F_{i, m}$ and the $F_{j, n}$; Here, the constraint correlation is mainly reflected as the distance requirement, position requirement and orientation requirement, etc.

For example, the initial requirement is the distance requirement between the surface $F_{1,1}$ and the $F_{1,3}$, as shown in Fig. 5.

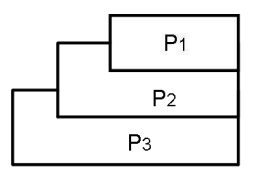

(a)

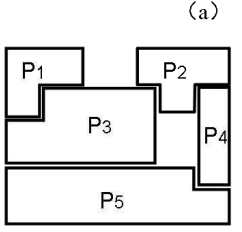

(c)
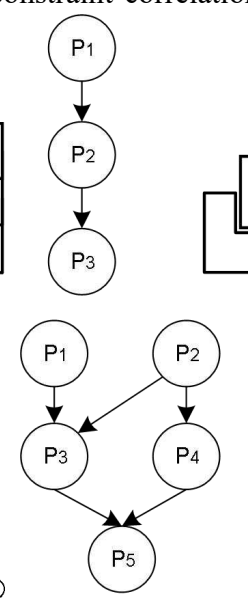

Fig. 4 Basic form of the transfer path of part-level

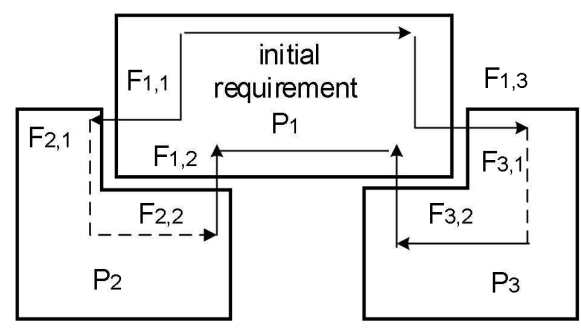

(a)

Fig. 5 Transfer path of surface-level

(2) Establish transfer path of part-level

(1) If $i=j$, that is, the surface $F_{i, m}$ and $F_{j, n}$ belong to the same part. The part $i$ is used as the start part to traverse the matching constraint relationship between the parts, and the transfer path of part-level is established.

(2) If $i \neq j$, part $i$ and part $j$ are set as the start part and end part respectively. The matching constraint relationship between the parts is traversed, and the transfer path of part-level is established. For example, there are two series paths of part-level, in Fig. 5a and Fig. 5b:

Path 1: $\mathrm{P}_{1} \rightarrow \mathrm{P}_{2}$

Path 2: $\mathrm{P}_{1} \rightarrow \mathrm{P}_{3}$

(3) Establish transfer path of surface-level

(1) Establishing transfer path of extended part-level (d)

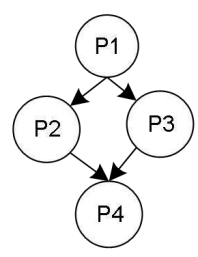

(b)
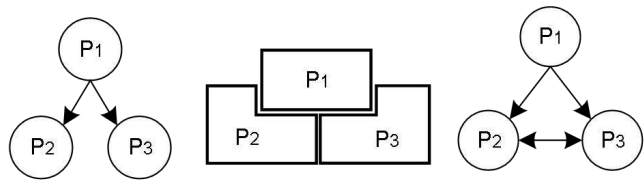

(e)

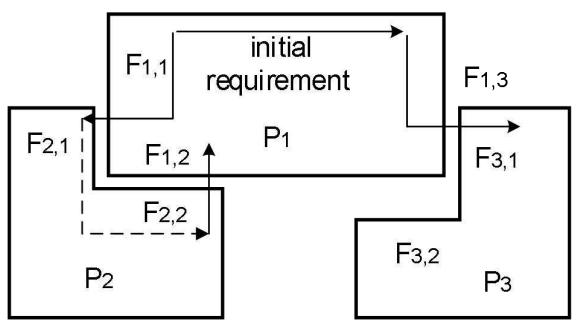

(b)

Based on the transfer path of part-level, the correlation information of the mating surface pair between the adjacent parts is added to the path, and the transfer path of extended part-level is obtained.

The path is shown in Fig. 5a :

Transfer path a1 of extended part-level:

$$
\mathrm{P}_{1} \stackrel{\mathrm{F}_{2,2} \stackrel{\mathrm{F}_{1,1} \stackrel{\mathrm{C}_{11,21}, 12}{\longrightarrow} \mathrm{F}_{2,1}}{\longrightarrow} \mathrm{F}_{1,2}}{\longrightarrow} \mathrm{P}_{2} \text {; }
$$

Transfer path a2 of extended part-level:

$$
\mathrm{P}_{1} \stackrel{\mathrm{F}_{1,3} \stackrel{\mathrm{C}_{13,31}}{\longrightarrow} \mathrm{F}_{3,2} \stackrel{\mathrm{C}_{32,1} 12}{\longrightarrow} \mathrm{F}_{1,2}}{\longrightarrow} \mathrm{P}_{3} ;
$$

(2) Establish transfer path of surface-level

In addition to the transfer between the mating surfaces of different parts, the constraint correlation between the general structural surfaces in the parts is 
also an important link in the transfer of design requirements. The spatial positioning constraint between the general structural surfaces in the transfer path of extended part-level is added to form a continuous path of complete geometric tolerance.

The spatial positioning constraint between the general structure surfaces is expressed as follows:

$$
F_{i m} \stackrel{L_{i m, i n}}{\longrightarrow} F_{i n}
$$

Where, $L_{i m, i n}$ refers to the spatial positioning relationship between the general structure surface $F_{i, m}$ and $F_{i, n}$ in the part $P_{i}$. Unlike the matching constraint relationship $C_{i s, j t}$ between the mating surfaces in the assembly joint, $L_{i m, i n}$ includes not only fitting constraints such as fit, coaxial, and tangent, but also positioning constraints such as vertical, parallel, and inclined.

Taking Fig. 5a as an example, the vertical constraint between the general structural surfaces $\mathrm{F}_{2,1} \stackrel{\mathrm{L}_{21,22}}{\longrightarrow} \mathrm{F}_{2,2}$ and $\mathrm{F}_{3,1} \stackrel{\mathrm{L}_{31,32}}{\longrightarrow} \mathrm{F}_{3,2}$ is an important link in the transfer of design requirements; Therefore, they are added to the transfer path between mating surfaces to obtain the complete transfer path of surface-level:

Transfer path b1 of surface-level:

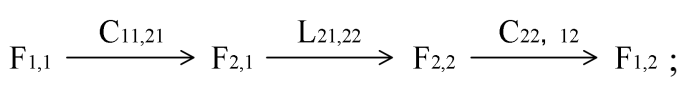

Transfer path b2 of surface-level:

$\mathrm{F}_{1,3} \stackrel{\mathrm{C}_{13,31}}{\longrightarrow} \mathrm{F}_{3,1} \stackrel{\mathrm{L}_{31,32}}{\longrightarrow} \mathrm{F}_{3,2} \stackrel{\mathrm{C}_{32,12}}{\longrightarrow} \mathrm{F}_{1,2}$;

(3) Analysis of transfer path of surface-level

Based on the above path, in order to clearly express the transfer process of geometric tolerance between parts, the complex transfer paths such as parallel, star and triangle paths are decomposed, that is, several independent series paths of part-level are obtained based on the expansion of common parts; On the basis, the matching constraints between the mating surfaces and the spatial positioning relationship of the general structure surface are added, and several transfer paths of surface-level are obtained.

Due to the existence of common mating surfaces, correlation often exists between the above-mentioned paths; In order to fully express the transfer process of geometric tolerances between related surfaces, it is necessary to combine those multiple transfer path of surface-level. That is, when the same surface exists in multiple transfer path of surface-level, it is used as a link to connect with the mating surfaces in the original path in sequence. As shown in Fig. 5a, $F_{1,2}$ form mating surface pairs with $F_{2,2}$ and $F_{3,2}$ in different paths respectively. Therefore, $F_{1,2}$ can be used as a common mating surface to establish the correlation between two series paths b1 and b2. In this process, the surface composition, the relationship between the surfaces and the transfer direction on the original paths $b 1$ and $b 2$ unchanged. The combined path is as follows:

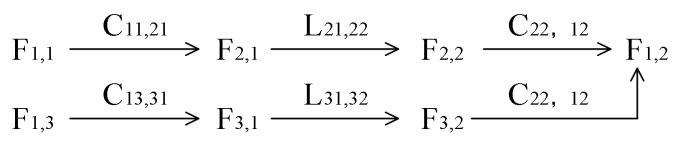

3.3 order of transfer paths for geometric tolerance items

For the same design requirement, when there are multiple transfer paths of part-level or surface-level, the ordering of transfer paths is important to the transfer process of geometric tolerance items. In order to determine the priority of multiple transfer paths, the following principles are proposed:

Principle 1: Principle of key part. Key part refers to the part that undertakes main functions and it is correlated with other parts in the path. For the part-level path, the priority of the path contain the key part is high; When the key parts exist in multiple paths, the path priority of the main function is the highest.

Principle 2: Principle of primary assembly joint. For several transfer paths of surface-level extended from the same part-level path, the transfer path contain the primary assembly joint has a higher priority

Principle 3: Principle of the shortest path. For transfer paths of part-level (or surface-level) that have the same start part (or surface) and end part (or surface), and they contain key parts and primary assembly joints as well, the path with fewer parts (or surfaces) has higher priority.

4 Generation and transfer of geometric tolerance 
items

4.1 Structural transformation of functional requirements

To ensure the realization of specific functions, multiple topological constraints often exist between product constituent units.

In the top-down design process, the function decomposition and reconstruction is carried out firstly. And then the geometric feature features can be generated according to the sub-function and the correlated constraints; Through this transformation of functional requirements to structural composition, the realization of product functions is guaranteed. This process is embodied in a hierarchical structure, as shown in Fig. 6. Firstly, based on the existing design

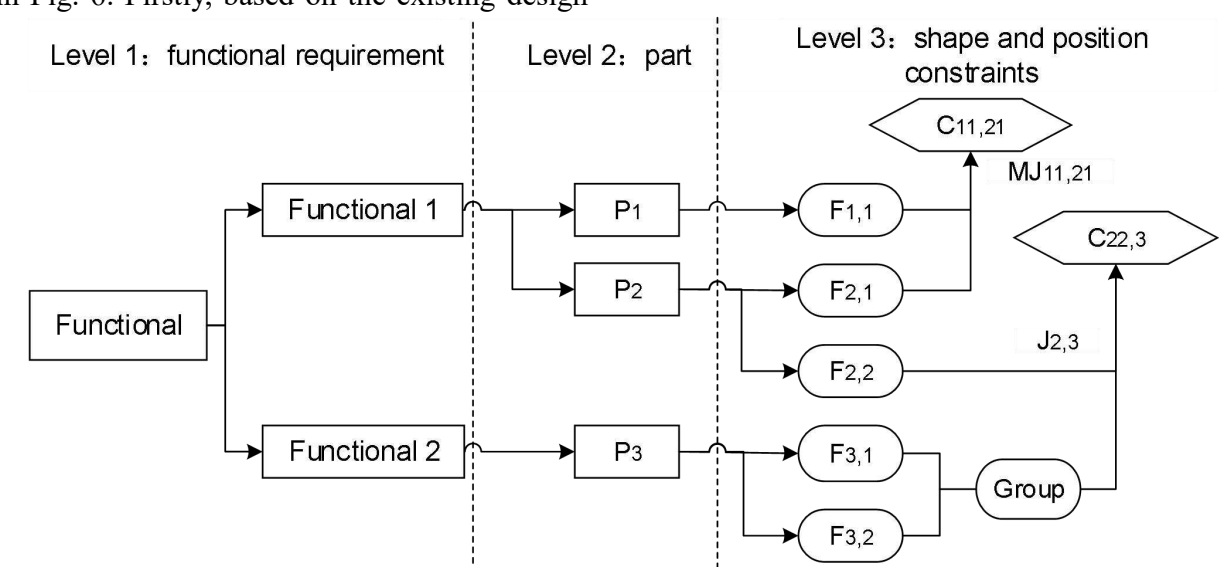

Fig. 6 Transformation Model of functional requirements

Based on the above process, the function constraint indirectly acts on the combination process of the sub-feature unit, which transforms the target function into the matching structure and the matching behavior. And then it is transformed into the shape and positioning constraint between the parts. These shape and positioning constraint can be further transformed into specific structural requirements such as distance, direction, positioning and shape between geometric features, thus the transformation from functional requirements to structural features and their correlations are fulfilled.

4.2 Generation of geometric tolerance items based on structural attributes

The design requirements are divided into functional requirements and structural constraints. The structural transformation method of functional requirements is given in 3.1, based on which the unified expression of knowledge, the function planning is carried out through multivariate conflict resolution to produce the functional combination of products or units. This is the first leve. Then, the functional planning scheme is extended to obtain the concept combination of parts to meet the functional requirements; On this basis, the geometric feature information implicit in the conceptual parts is extracted to form the functional structure attributes between the geometric feature elements. This is the second level. Finally, the shape and position constraints between the abstract physical structures are defined, and the transformation relationship between function and geometric feature is established. This is the third level.

functional requirements and structural constraints can be realized. On this basis, the generation of geometric tolerance items can be realized based on the association specification between geometric tolerance items and structural attributes.

During design process, the product structure attributes that directly affect the generation of geometric tolerance items mainly include the structural correlation between the parts or the surface, as well as the attributes of the assembly joints.

4.2.1 Generation of geometric tolerance items based on design requirements

The structure type of surface and its constraint type determine its corresponding geometric tolerance item. The commonly used surfaces in engineering are mainly planes and cylinders. While the constraint types between surfaces based on functional decomposition mainly include distance requirements, 
orientation requirements, positioning requirements and shape requirements. Based on the above surface requirements, the specifications for geometric tolerance generation are shown in Table 3.

types and the constraint types, that is design

Table 3 Specification for geometric tolerance generation based on design requirements

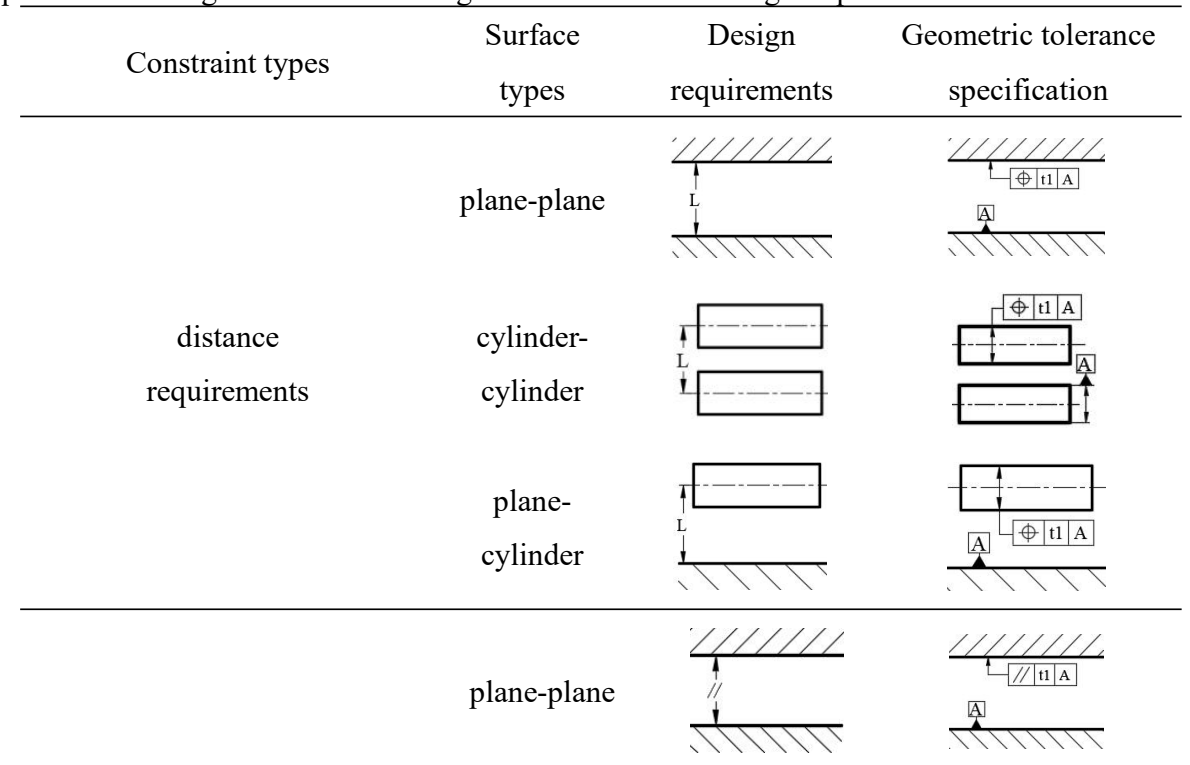

parallel

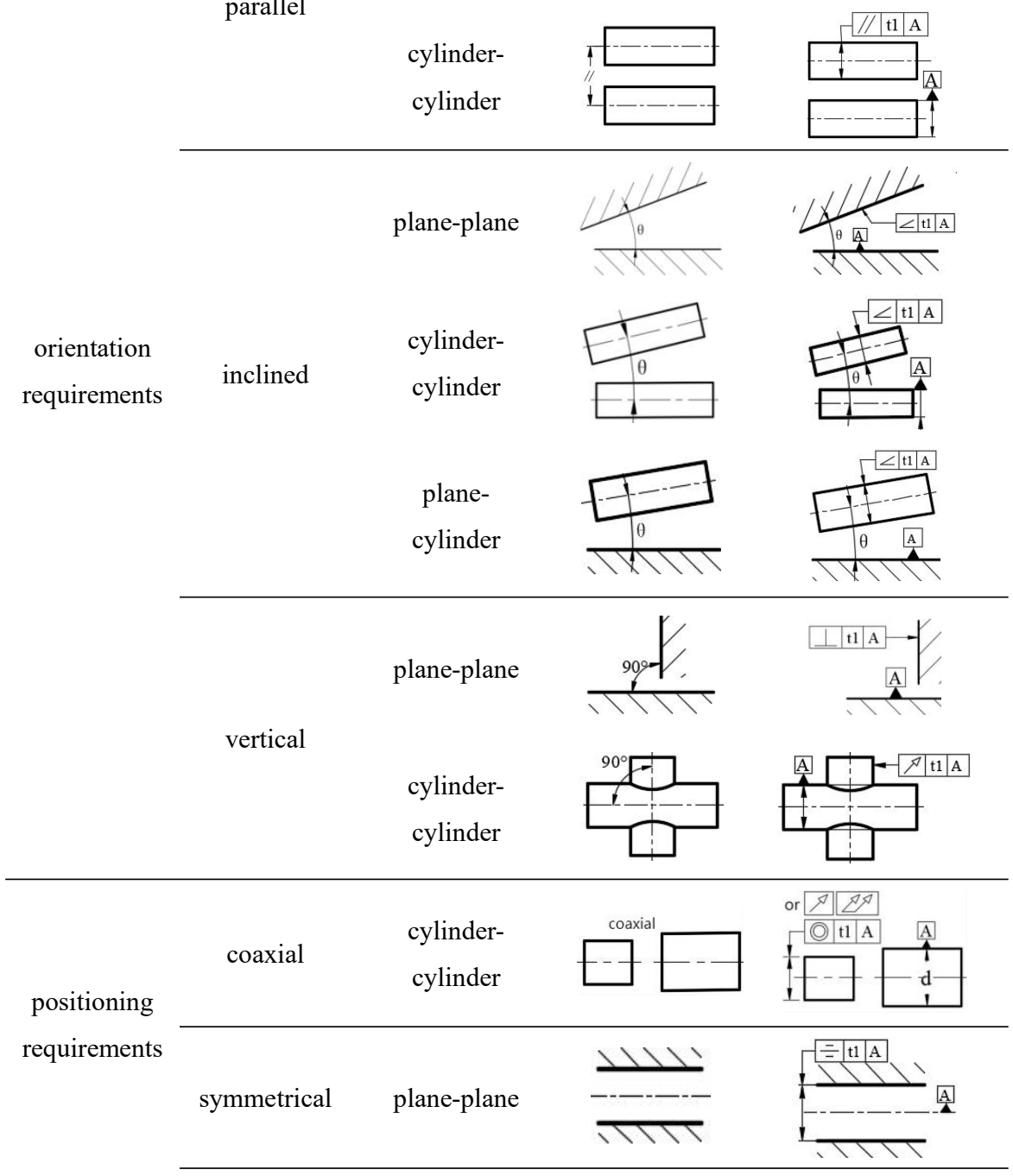




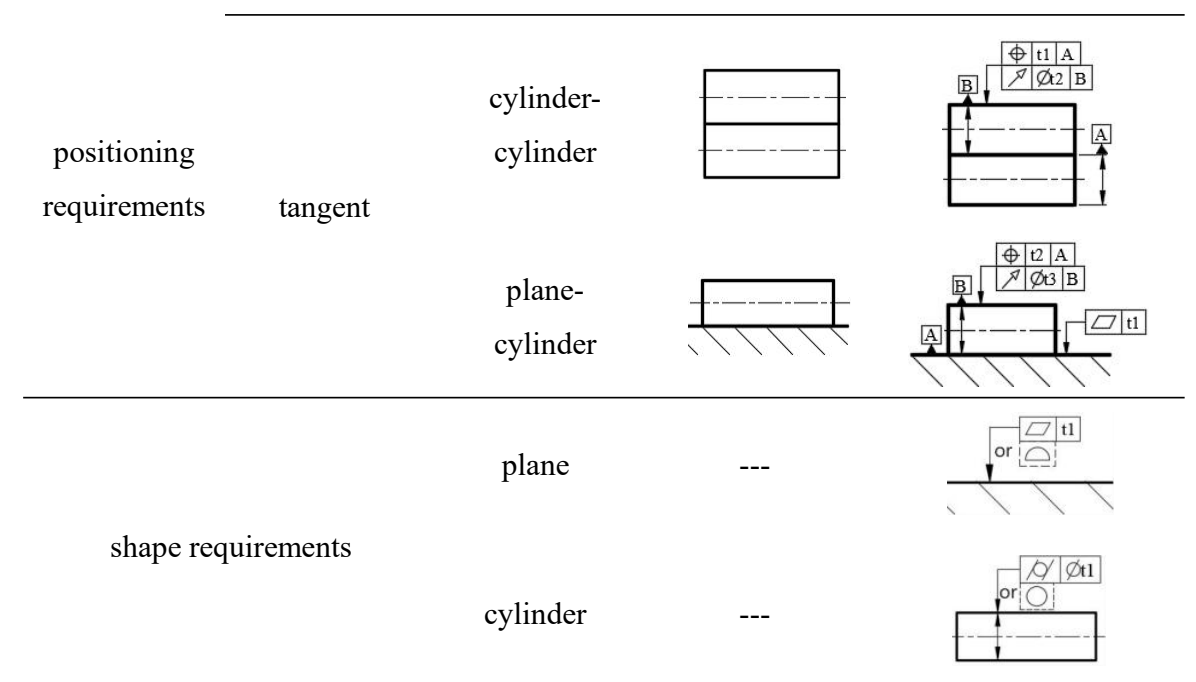

\subsubsection{Generation of geometric tolerance items based on assembly joint attribute}

According to attribute information such as the surface type, constraint requirements and priority order that compose the assembly joint, the generation specification of geometric tolerance items based on assembly joint attribute can be constructed.

For the meta-assembly joints (as MJ in Table 4) that constitute the composite assembly joint:

(1) When the meta-assembly joint is the primary assembly joint, the geometric tolerance items of the mating surface are self-reference tolerances such as profile tolerance or shape tolerance;

(2) When the meta-assembly joint is the secondary assembly joint, the mating surface of the primary assembly joint is used as the reference. And the geometric tolerance items are cross-reference tolerances, that is, direction tolerance, positioning tolerance or profile tolerance. Specific tolerance items can be derived according to the surface type and design requirements, as shown in Table 3;

(3) When the meta-assembly joint is the third assembly joint, the matching surface of the primary assembly joint is the first reference, the matching surface of the secondary assembly joint point is the second reference. The geometric tolerance items are cross-reference tolerances

For the composite assembly joints (as $\mathrm{J}_{1} \sim \mathrm{J}_{6}$ in Table 4):

(4) Firstly, the positioning constraints should be considered. The geometric tolerance items of the matching surfaces are same, when the spatial positioning constraint between the meta-assembly joints is consistent with the type of matching constraints between the mating surfaces. And when the two constraints are inconsistent, the positioning constraints between the meta-assembly joints should be guaranteed first, and then the constraints between the mating surfaces.

(5) On the basis of (4), according to (1)(2)(3), the geometric tolerance items of the meta-assembly joint forming the composite assembly joint.

For example, for the composite assembly joint " $J_{1}=M J_{1} \stackrel{f i t}{\longrightarrow} M J_{2}$ " in Table 4, the positioning constraint type between the meta-assembly joint $\mathrm{MJ}_{1}$ and $\mathrm{MJ}_{2}$, and the matching constraint type between the two mating surfaces of $\mathrm{MJ}_{1}$ and $\mathrm{MJ}_{2}$ are all plane fit. So the geometric tolerance items are all flatness. On the other hand, for the composite assembly joint " $J_{2}=M J_{1} \stackrel{\text { parallel }}{\longrightarrow} M J_{2}$ " in Table 4, the positioning constraint type between the meta-assembly joints $\mathrm{MJ}_{1}$ and $\mathrm{MJ}_{2}$ is parallel, and the matching constraint type between the two mating surfaces forming $\mathrm{MJ}_{1}$ and $\mathrm{MJ}_{2}$ is fit. Therefore, priority is given to ensure that the position constraints between the meta-assembly joints are parallel. When the meta-assembly joint is used as the primary assembly contact, the surface profile not only ensures the shape of surface, but also restricts the position between the surfaces. So the surface profile can be selected. When the meta-assembly joint is used as the secondary assembly contact or the third assembly contact, the cross-reference tolerance is selected according to the position relationship between the surfaces. 
Table 4 Specification for geometric tolerance generation of assembly joint

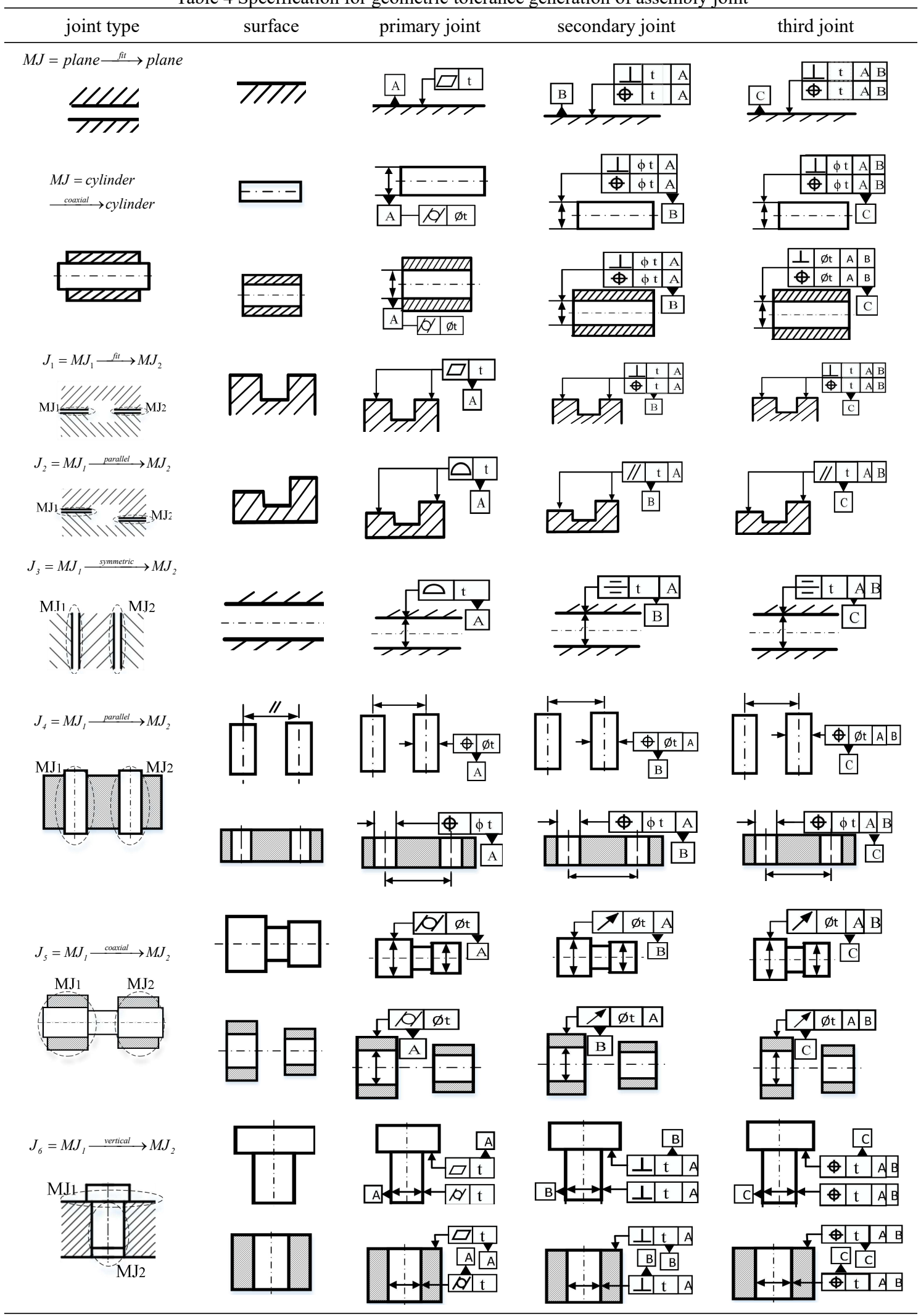

4.2.3 Datum transfer based on assembly joint

In the design process of geometric tolerance items, with the decomposition of the original functional requirements, the geometric tolerance items will be gradually decomposed to the part and the surface. In this process, the datum will also gradually migrate from the product level to the part-level and the surface-level with the decomposition of the assembly 
joints. During this process, two specifications should be followed:

Specification 1: When the initial datum is one of the mating surface constructing the assembly joint, the datum will be transferred to another mating surface with the decomposition of the assembly joint, as shown in Fig. 7a. Among them, when the types of the two mating surfaces are the same and the transferred datum and the initial measured element belong to the same part, the geometric tolerance items of the measured element relative to the datums are consistent with the initial tolerance items; When the types of the two mating surfaces are inconsistent, that is, the type of the datums changes after the transfer, the geometric tolerance items of the initial measured element relative to the reference datums selected according to Table 3. At the same time, in order to ensure the accuracy of geometric tolerance transmission, the datum is added with shape and position constraints, and its geometric tolerance items need to be determined according to the type and priority of the assembly joint, according to Table 4 .

As shown in Fig. $7 \mathrm{~b}$, according to structural transformation of the original functional requirements, the surface $F_{1,1}$ of $P_{1}$ has a positioning requirement, taking the surface $F_{2,1}$ of $P_{2}$ as the datum. The surface $F_{2,1}$ and $F_{1,2}$ cooperate with each other to form the primary assembly joint, and the surface $F_{2,2}$ and $F_{1,3}$ form the secondary assembly joint. With the transfer of geometric tolerances, the datum migrate from $\mathrm{F}_{2,1}$ to $\mathrm{F}_{1,2}$ inside the assembly joint. According to the above specifications, the original measured element $F_{1,1}$ still maintains the position tolerance requirements relative to the new datum $F_{1,2}$ in the same part. To ensure the accuracy of tolerance transmission, according to Table 4 , the geometric tolerance items of the mating surfaces $F_{1,2}$ and $F_{2,1}$ of the primary assembly joint are flatness; the geometric tolerance items of the mating surfaces $F_{1,3}$ and $F_{2,2}$ of the secondary assembly joint are perpendicularity with the primary joint surface as the datum.

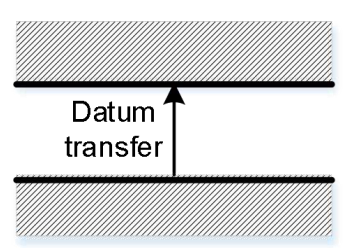

$M J_{1}$

(a)

Fig. 7 Datum transfer based on assembly joint 1

Specification 2: When the initial datum is not one of the mating surface, the orientation and positioning requirements of the original measured element relative to the original datum are converted into the following three geometric tolerance requirements:

(1) In the part where the original measured element is located, the orientation and positioning requirements of the initial measured element relative to the new datum in the same part;

(2) The shape and positioning requirements of the mating surfaces;

(3) In the part where the original datum is located, the direction and positioning requirements of the mating surface relative to the original datum.

As shown in Fig. 8, the initial design requirement is the positioning requirement of the measured element $F_{1,1}$ relative to the datum $F_{2,3}$, where the initial datum $F_{2,3}$ does not form an assembly joint with any surface of the part $P_{1}$. With the transfer of geometric tolerances, the initial geometric tolerances will be decomposed. In $\mathrm{P}_{1}$ where the measured element is located, $F_{1,1}$ should have positioning requirements relative to the new datum $\mathrm{F}_{1,2}$; In the part $\mathrm{P}_{2}$, the mating surface $F_{2,1}$ constituting the primary assembly joint is relative to the original reference $F_{2,3}$ to ensure the positioning requirement. In the main assembly joint formed by the mating surfaces $F_{2,1}$ and $F_{1,2}$, and the secondary assembly joint formed by $F_{2,2}$ and $F_{1,3}$, the flatness and perpendicularity tolerance items consistent with Fig. 7 are generated according to Table 
primary assembly joint is located should have a
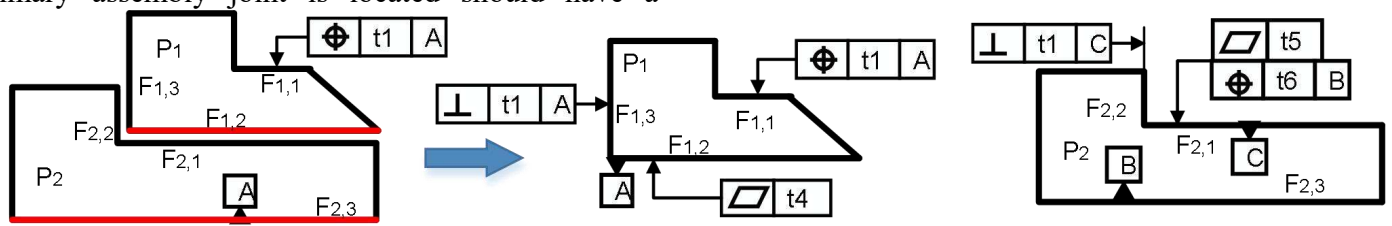

Fig. 8 Datum transfer based on assembly joint 2

5 The transfer process of geometric tolerance items based on assembly joints

The top-down transfer process of geometric tolerance items is shown in Fig. 9. Taking the initial design requirements as the start point, under the constraints of functional requirements and structural associations, the initial geometric tolerance items are transferred from the product layer to the part layer, finally to the geometric surface layer along the part-level and surface-level transfer path. The process is mainly include the following 6 steps:

(1) Determination of initial geometric tolerance item

According to the design requirements and structural constraints of the product, the initial geometric tolerance items are determined refer to Table 3;

(2) Accomplish the structural transformation of functional requirements

Parts and surfaces are analyzed from the aspect of functional requirements, function correlations are extracted, based on which the structural transformation models of functional requirements are constructed, and structural transformation of functional requirements is realized;

(3) Determine the order of assembly joints

According to the mating surface composition of the assembly joints and the type of constraint correlation, the degree of freedom analysis is performed to determine the order of the assembly joints;

(4) Construct the transfer path of geometric tolerance items

(1) Construct the transfer path of part-level

Take the two parts associated with the initial design requirements as the start part and the end part of the path respectively, or the same part required by the initial design requirements as the start part; And then take the parts with related functions or structural connections as the nodes, and follow the sequence of upper part to lower part, part-level geometric tolerance transfer path can be generated. Finally, the paths are ordered.

(2) Construct the transfer path of surface-level

Based on the functional requirements and structural constraints of the mating surfaces, as well as the shape and positioning constraints between the general structural surfaces, the transfer paths of surface-level are generated and ordered;

(5) Transfer of geometric tolerance items based on structural constraints

(1) Selection of geometric tolerance items based on structural constraints

Based on the shape and positioning constraints between the functional elements and the structural constraints between the surfaces, the geometric tolerance items of the start part are selected;

(2) Transfer of datum

According to the position of the datum, the datums are transferred between assembly joints and the general structure surface respectively. The positioning tolerance is added to the parts where the datums are located, and the shape tolerance is added to the mating surface to ensure the matching accuracy;

(3) Path-based geometric tolerance items transfer

The geometric surface where the initial design requirements are located is used as the start surface, and the geometric tolerance items are transferred in the direction of the geometric tolerance transfer path. The geometric tolerance items are transferred sequentially to the related surfaces of the associated parts until the surface is the geometric surface 
corresponding to the initial design requirements in the end part.
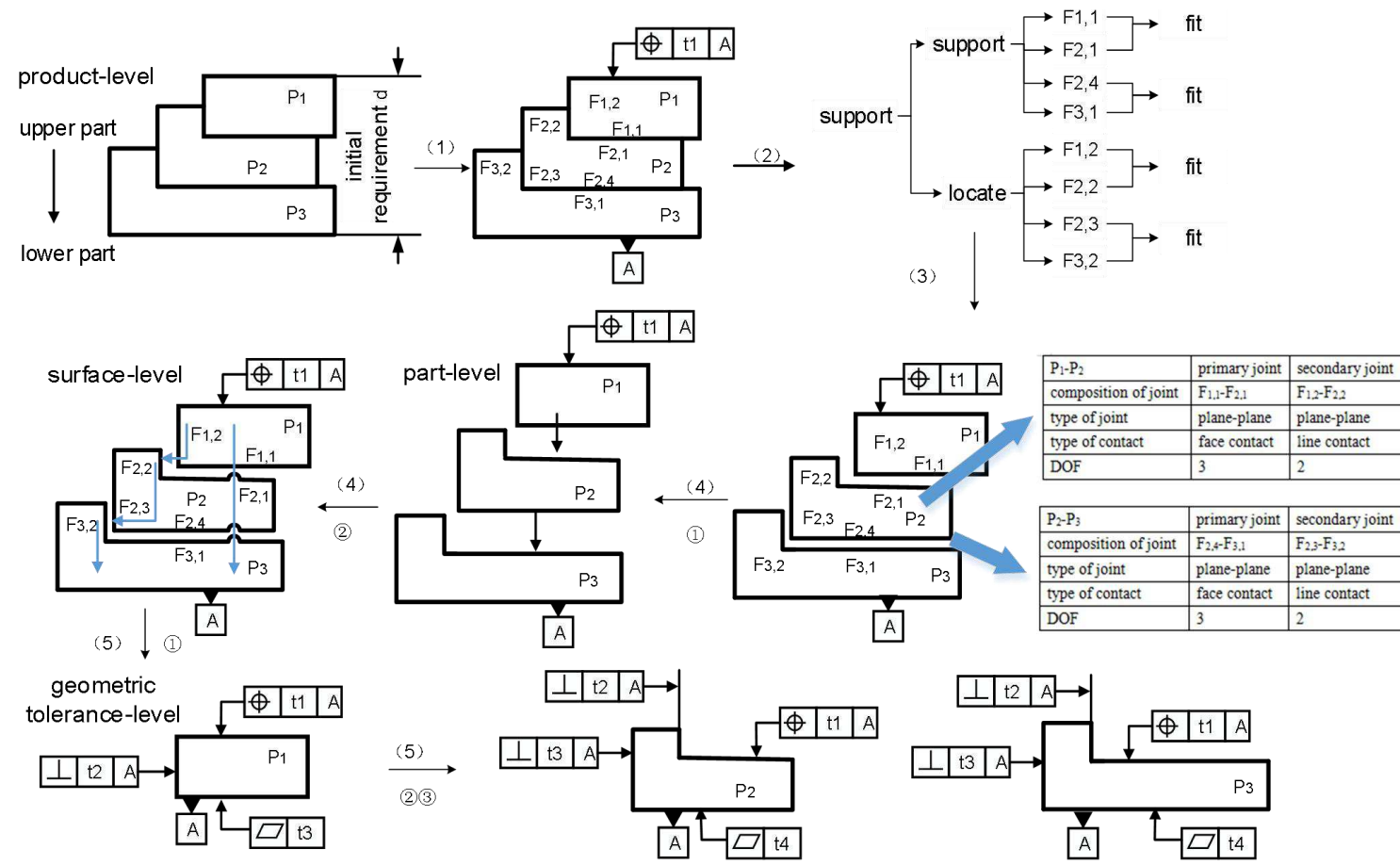

Fig. 9 Transfer process of geometric tolerance items based on assembly joints

\section{Example}

The transmission parts and connection parts in the crankshaft-piston mechanism are taken as examples to verify the top-down transfer method of tolerance items above-mentioned. The crankshaft-piston mechanism converts the reciprocating motion of the piston into the rotational motion of the crankshaft, and at the same time converts the pressure on the piston into the torque output by the crankshaft, so as to drive the load. The mechanism is mainly composed of piston, connecting rod group and crankshaft, as shown in Fig. 10.

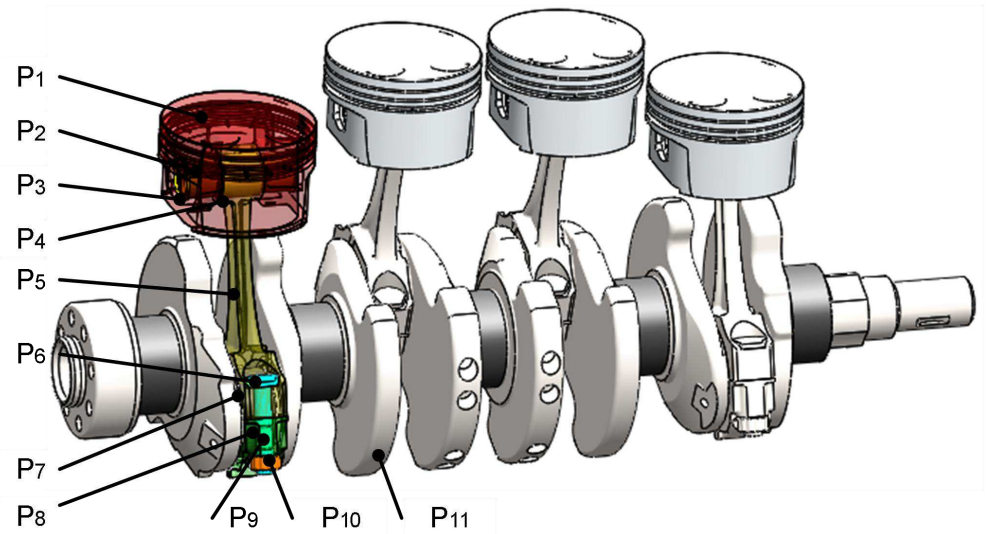

$\mathrm{P}_{1}$-piston, $\mathrm{P}_{2}$-piston pin, $\mathrm{P}_{3}$-retaining ring, $\mathrm{P}_{4}$-rod bushing, $\mathrm{P}_{5}$-connecting rod, $\mathrm{P}_{6}$-bolt, $\mathrm{P}_{7}$-bearing bush 1 , $\mathrm{P}_{8}$-bearing bush 2, $\mathrm{P}_{9}$-connecting rod cover, $\mathrm{P}_{10}$-nut, $\mathrm{P}_{11}$-crankshaft

Fig. 10 Crankshaft piston mechanism of engine

Among them, the piston pin $\mathrm{P}_{2}$, the retaining ring $\mathrm{P}_{3}$, the bolt $\mathrm{P}_{6}$ and the nut $\mathrm{P}_{10}$ are standard parts. So there is no need for geometric tolerance analysis for them.

The allocation process of the mechanism's geometric tolerance items is as follows:
(1) According to the functional requirements and structural constraints of the crankshaft-piston mechanism, the initial geometric tolerance items are determined.

In order to avoid defects such as piston pulling cylinder, abnormal wear of main bearing, increased fuel consumption, etc., and to improve the mechanism's service 
life. The parallelism error of piston pin holes, connecting rod journals, crankshafts and other components should be controlled effectively. Therefore, the initial design requirement of the crankshaft-piston mechanism is that the pin hole axis $l_{l}$, the rod journal axis $l_{2}$, and the crankshaft journal axis $l_{3}$ remain parallel. Refer to Table 3, the initial geometric tolerance items of related parts can be determined, as shown in Fig. 11;

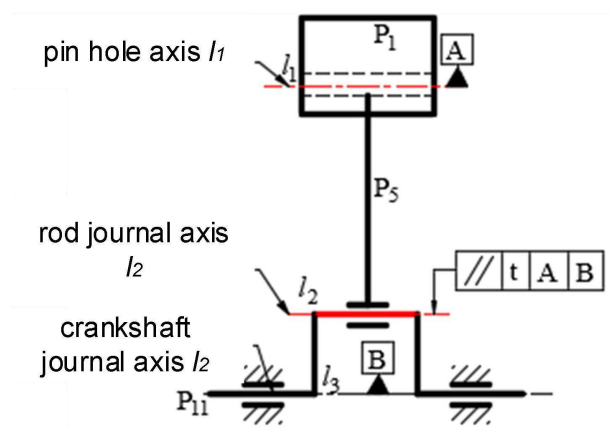

Fig. 11 Transformation of initial design requirements to geometric tolerance items

(2) Structural transformation of functional

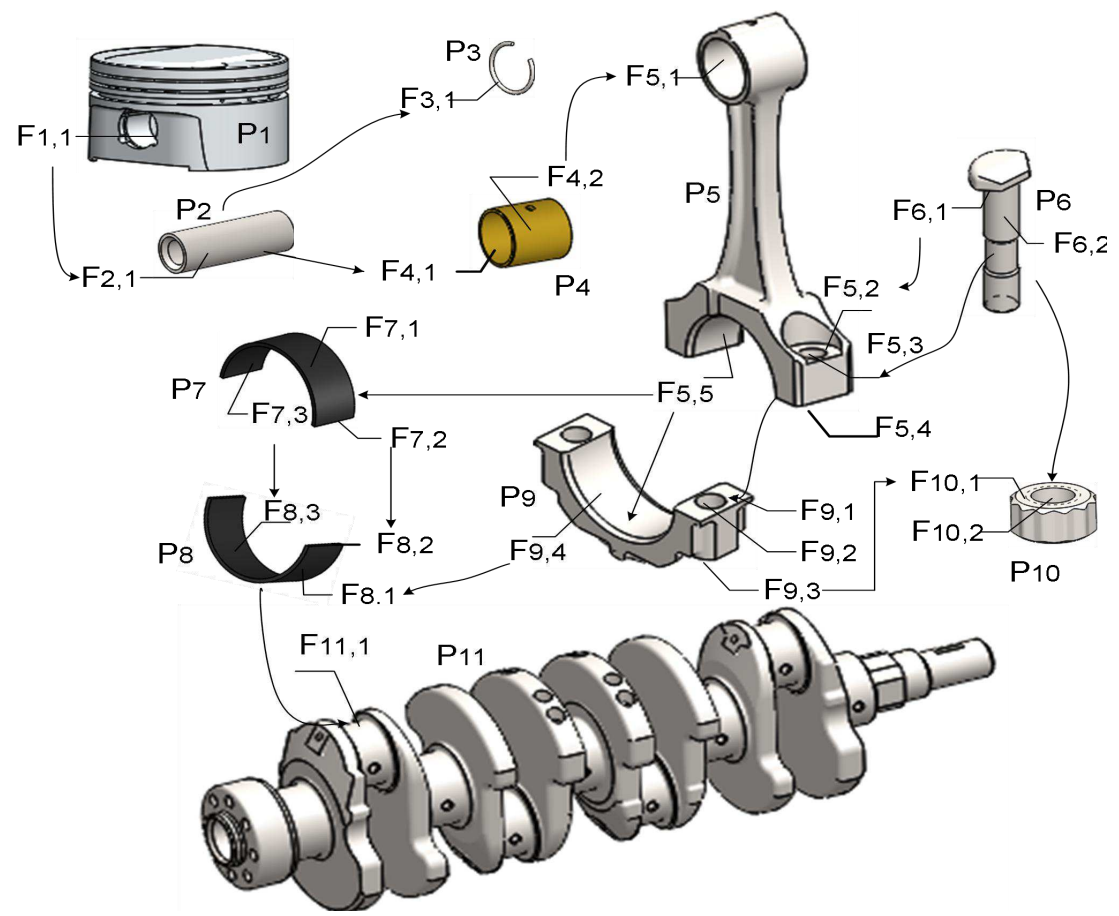

Fig. 12 The functional surface of the crankshaft-piston mechanism and the correlation model

(3) Determine the order of assembly joints

Analyze the composition of the mating surfaces of all the assembly joints between the transmission parts and the connection parts in the crankshaft-piston mechanism. And analyze the types of constraint associations of all the assembly joints. According to the number of freedom of the assembly joint, the order requirements.

According to the functional characteristics and structural constraints, the functional surface of each part in the crank-piston mechanism is extracted, as shown in Fig. 12.

Based on the function decomposition, the mechanism includes two main functions: transmission and connection. Among them, the transmission function is mainly realized by the parts $\mathrm{P}_{1}, \mathrm{P}_{2}, \mathrm{P}_{5}, \mathrm{P}_{9}$, and $\mathrm{P}_{11}$. The connection function is undertaken by the parts $\mathrm{P}_{6}, \mathrm{P}_{5}, \mathrm{P}_{9}$, and $\mathrm{P}_{10}$. Take transmission function and connection function as examples. Firstly, the transmission and the connection function requirements are transformed into the spatial positioning relationship between the surfaces, and the shape and positioning constraints are established, based on which the structural transformation of functional requirements is realized. The main function transformation model of the crankshaft-piston mechanism is shown in Fig. 13. 
part respectively, and other transmission parts are the intermediate nodes. The transfer path of part-level geometric tolerance item based on the transmission function, named path I, is constructed. Similarly, transfer paths II and III based on the connection function can be established. Since parts $\mathrm{P}_{6}$ and $\mathrm{P}_{10}$ in path III are standard parts, path III can be ignored.

$$
\begin{aligned}
& \text { I } \quad \begin{array}{l}
\mathrm{P}_{1} \longrightarrow \mathrm{P}_{2} \longrightarrow \mathrm{P}_{5} \longrightarrow \mathrm{P}_{11} \\
\text { II } \quad \mathrm{P}_{6} \rightarrow \mathrm{P}_{5} \rightarrow \mathrm{P}_{9} \rightarrow \mathrm{P}_{10}
\end{array} \\
& \text { III } \quad \mathrm{P}_{6} \rightarrow \mathrm{P}_{10} ;
\end{aligned}
$$

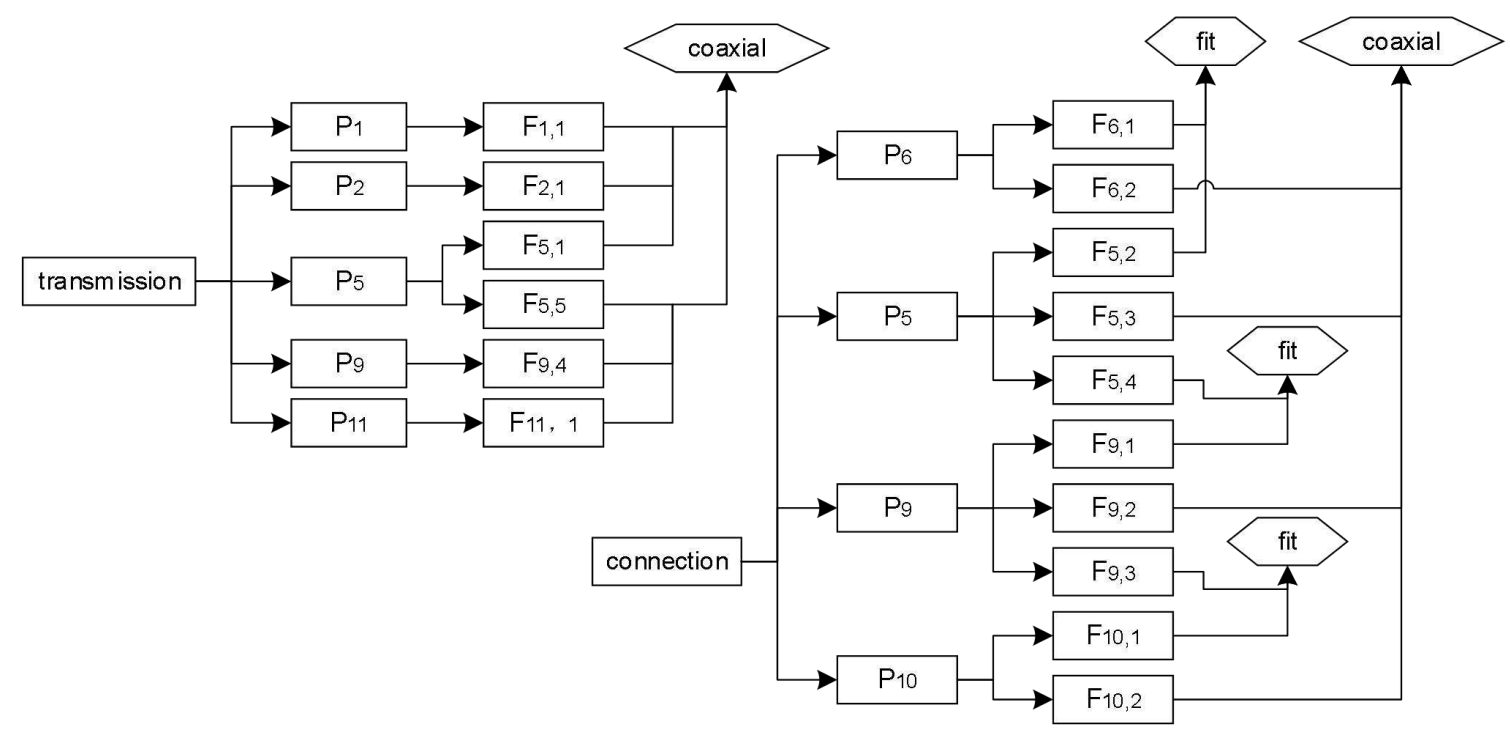

Fig. 13 Structural transformation model of functional requirement

Table 5 Analysis of the assembly joints

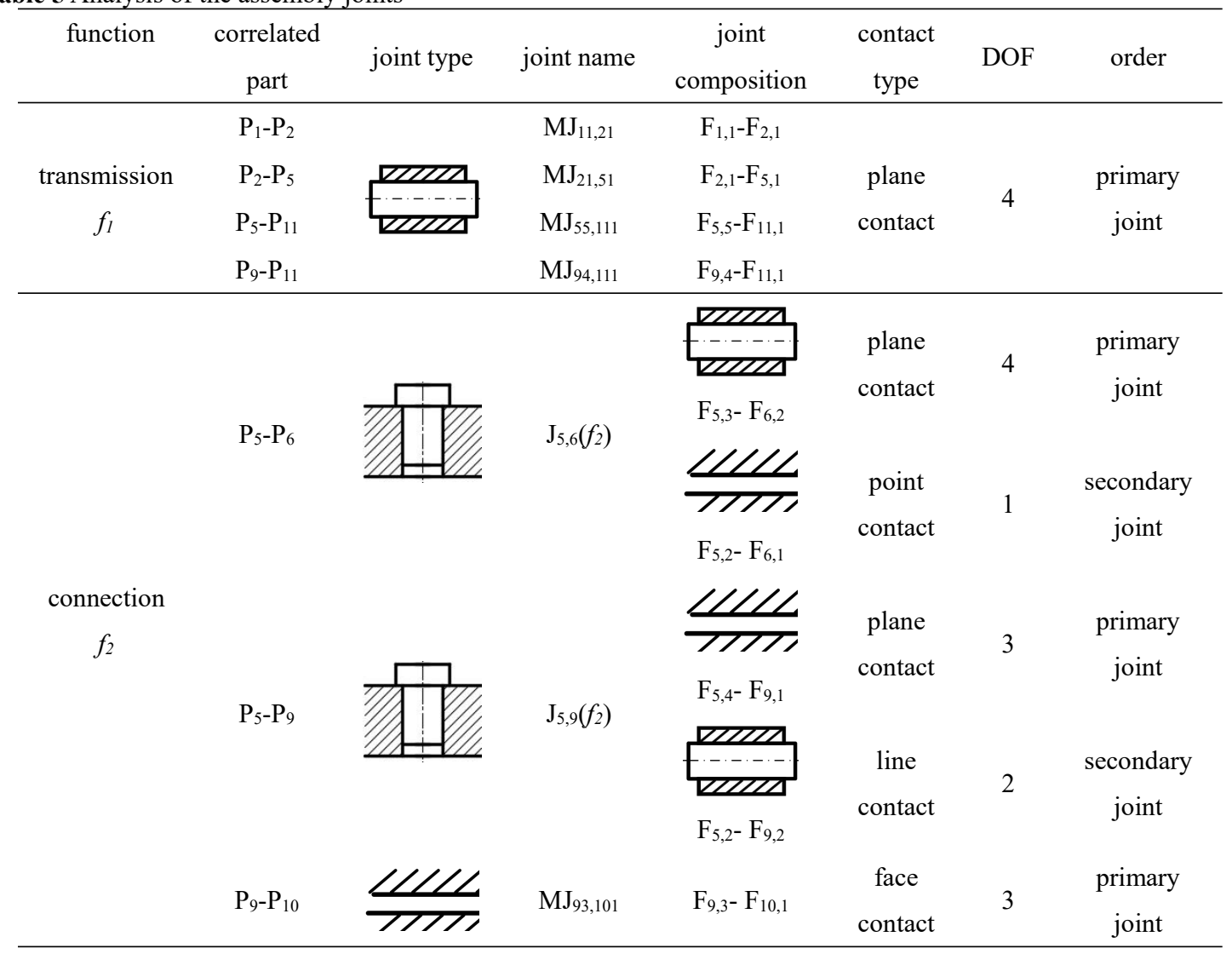

(2) Construct the transfer path of extended part-level
In the part-level paths I and II, the related information of mating surface pairs between adjacent 
parts are added to obtain the transfer paths of extended part-level named path a and path $\mathrm{b}$.

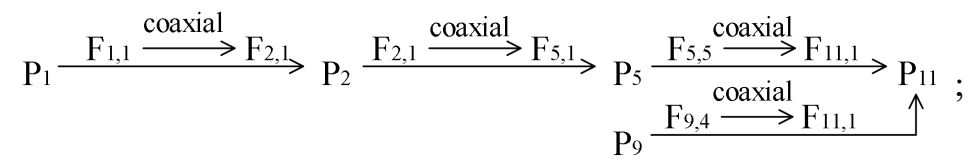

b

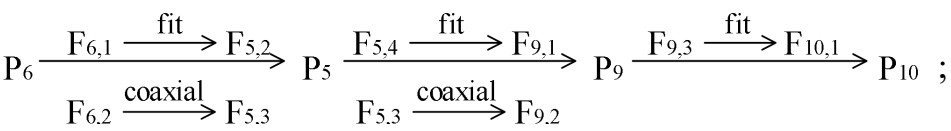

(3) Construct the transfer path of surface-level

The extended part-level path $\mathrm{a}$ and $\mathrm{b}$ are further represented graphically. And then, the positioning constraint between the general structure surface in the part is added, based on which the graphical representation of the surface-level transfer path is obtained. As shown inFig. 14a, in order to ensure transmission accuracy, the axis of hole $F_{5,1}$ need to be parallel to the axis of hole $\mathrm{F}_{5,5}$, therefore, the position

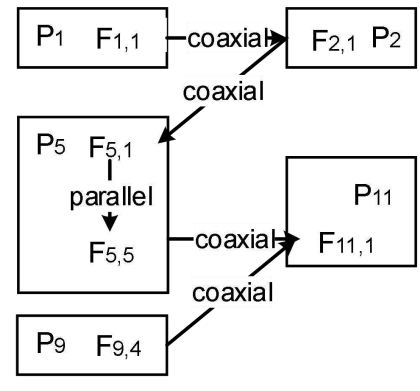

(a) constraint 'parallel' is added between general structural planes $F_{5,1}$ and $F_{5,5}$. As shown inFig. $14 \mathrm{~b}$, since the two meta-assembly joints composing the composite assembly joint $J_{5,6}(\mathrm{f} 2)$ should be vertical according to the design requirement, 'vertical' constraint should be added between the general structural surfaces $\mathrm{F}_{5,3}$ and $\mathrm{F}_{5}, 2$ that constitute the joint. Similarly, 'vertical' constraints should be added between the surfaces $F_{9,2}$ and $\mathrm{F}_{9,3}$

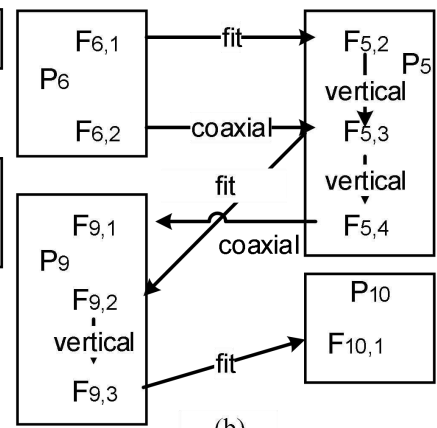

(b)

Fig. 14 Graphical representation of the surface-level transfer paths

Based on the above-mentioned result, the surfaces are sequentially connected according to the initial transfer direction, and the continuous transfer paths of surface-level i, ii, iii are obtained:

$$
\begin{aligned}
& \mathrm{i}_{1,1} \stackrel{\text { coaxial }}{\longrightarrow} \mathrm{F}_{2,1} \stackrel{\text { coaxial }}{\longrightarrow} \mathrm{F}_{5,1} \stackrel{\text { parallel }}{\longrightarrow} \mathrm{F}_{5,5} \stackrel{\text { coaxial }}{\longrightarrow} \mathrm{F}_{11,1} \text {. } \\
& \mathrm{F}_{9.4} \text { coaxial } \uparrow \\
& \text { ii } \mathrm{F}_{6,2} \stackrel{\text { coaxial }}{\longrightarrow} \mathrm{F}_{5,3} \stackrel{\text { vertical }}{\longrightarrow} \mathrm{F}_{5,4} \stackrel{\text { fit }}{\longrightarrow} \mathrm{F}_{9,1} \text {; } \\
& \text { iii } \mathrm{F}_{6,1} \stackrel{\text { fit }}{\longrightarrow} \mathrm{F}_{5,2} \stackrel{\text { vertical }}{\longrightarrow} \mathrm{F}_{5,3} \stackrel{\text { coaxial }}{\longrightarrow} \mathrm{F}_{9,2} \stackrel{\text { vertical }}{\longrightarrow} \mathrm{F}_{9,3} \stackrel{\text { fit }}{\longrightarrow} \mathrm{F}_{10,1} \text {; }
\end{aligned}
$$

Analyzing the priority order of each meta-assembly joint that constitutes the composite assembly joint, and then the multiple surface-level paths generated under the same functional requirement are ordered. The part-level path $\mathrm{b}$ includes two composite assembly joints $\mathrm{J}_{5,6}(\mathrm{f} 2)$ and $J_{5}, 9(f 2)$. According to the principle of primary assembly joint, the mating surfaces $F_{6,2}, F_{5,3}$ forming the primary assembly joint have priority over $F_{6,1}, F_{5,2}$, and the mating faces $F_{5,4}, F_{9,1}$ forming the primary assembly joint have priority over $F_{9,2}, F_{5,2}$. So the path ii through the surface $F_{5,3}, F_{5,4}, F_{9,1}$ with $F_{6,2}$ as the start surface has a high priority.

(5) Transfer of geometric tolerance items based on structural constraints

(1) Selection of initial tolerance items in the path

Firstly, the geometric tolerance items are determined for the initial surface (non-standard parts) of each surface-level transfer path. When the initial surface is a mating surface constitutes the assembly joint, the geometric tolerance items can be selected according to 
the function requirements, surface types and the order of assembly joints by referring to Table 3 and Table 4 . When the initial surface is a general structural surface, the geometric tolerance items can be selected according to its functional requirements, surface types, the shape and positioning constraints by referring to Table 3 .

In this example, the mating surface $F_{1,1}$ is the initial surface of the transfer path $i$, the primary assembly joint $\mathrm{J}_{11,21}$ is formed by $\mathrm{F}_{1,1}$ and $\mathrm{F}_{2,1}$, and the design requirement is that the two cylinders $F_{1,1}$ and $F_{2,1}$ are coaxial. Therefore, the mating surfaces $F_{1,1}$ and $F_{2,1}$ must have cylindricity tolerance requirements, referring to Table 4; Similarly, the geometric tolerance item of the initial surface $F_{5,3}$ of path ii is cylindricity. The mating surface $F_{5,2}$ is the initial surface of the path iii, which forms the secondary assembly joint $J_{52,61}$ with $F_{6,1}$. And the design requirement is that $F_{5,2}$ and $F_{6,1}$ fit together, so the geometric tolerance item is 'perpendicularity' with the mating surface $F_{5,3}$ of primary assembly joint as the datum. The geometric tolerance items corresponding to the initial surface in each surface-level transfer path are shown in Table 6.

(2) Transfer of datum

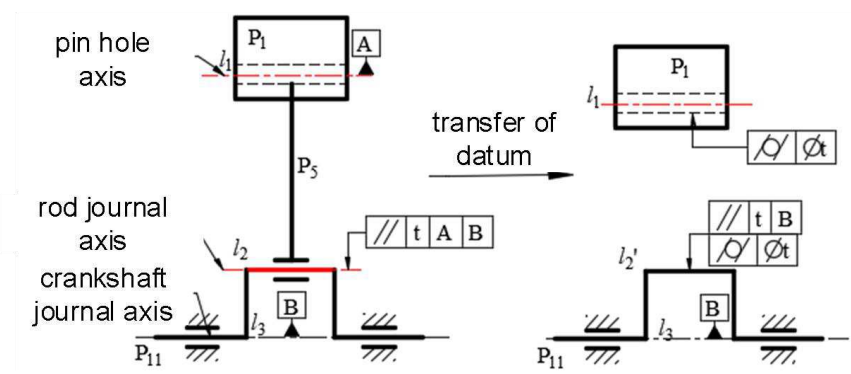

In the process of generating geometric tolerance items for the crankshaft-piston mechanism, the spatial positioning constraints between the parts under the initial design requirements will gradually be transformed into the shape and positioning constraints between the geometric feature in the parts. This process is bound to be accompanied by the transfer of datums.

As shown in Fig. 15, one of the generated initial geometric tolerance items is the parallelism tolerance of axis $l_{2}$ relative to axis $1_{3}$; Since $F_{11,1}$ and $F_{5,5}$ cooperate with each other to form assembly joint, the assembly-level tolerances will be converted into part-level tolerances during the top-down design process of geometric tolerances. That is, the parallelism tolerance will be converted into the parallelism of the crankshaft axis $l_{2}^{\prime}$ with respect to the axis $l_{3}$, which is coaxial with the axis $1_{2}$ in $\mathrm{P}_{11}$. At the same time, in order to ensure the matching accuracy, the tolerance item 'cylindricity' is added to the mating surfaces $F_{11,1}$ and $\mathrm{F}_{5,5}$.

Table 6 Initial tolerance items for each path

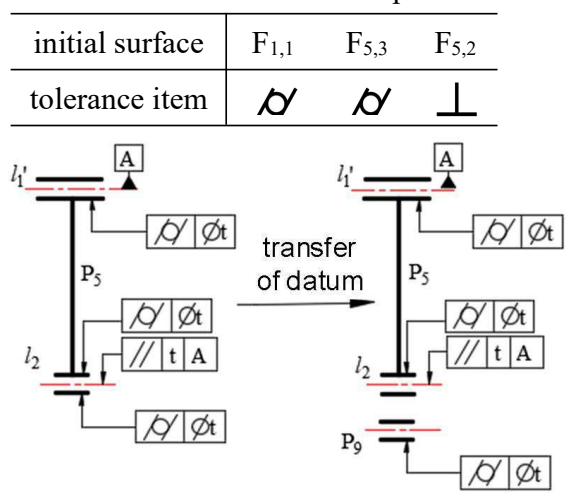

Fig. 15 Transfer of datums under the initial design requirement

(3) Path-based geometric tolerance items transfer

Taking the initial surface of the transfer path as the start point, the geometric tolerance items can be transferred along the surface-level path. When two adjacent surfaces in the path form an assembly joint, if the type of the matching surfaces are same, the geometric tolerance items will be copied and transferred. If the type of the matching surfaces are different, the geometric tolerance items are determined according to the shape and positioning constraints between the surfaces (see Table 3). When two adjacent surfaces in the path are the general structural surfaces of the same part, the geometric tolerance items are transferred according to their shape and positioning constraints (see Table 3). In this process, if the mating surface belong to a standard part, it is only taken as the transfer node and no tolerance item is assigned to it.

The surface-level transfer path of the crankshaft piston mechanism is shown as follows:

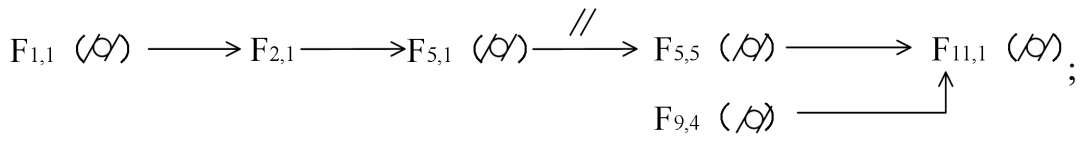




$$
\begin{aligned}
& \mathrm{F}_{6,2} \longrightarrow \mathrm{F}_{5,3}(\mathrm{O}) \stackrel{\perp}{\longrightarrow} \mathrm{F}_{5,4}(\square) \longrightarrow \mathrm{F}_{9,1}(\square) ; \\
& \mathrm{F}_{6,1} \longrightarrow \mathrm{F}_{5,2} \stackrel{\perp}{\longrightarrow} \mathrm{F}_{5,3}(\mathrm{O}) \longrightarrow \mathrm{F}_{9,2} \stackrel{\perp}{\longrightarrow} \mathrm{F}_{9,3}(\square) \longrightarrow \mathrm{F}_{10,1} ;
\end{aligned}
$$

$F_{2,1}, F_{6,2}, F_{6,1}$ and $F_{10,1}$ in the above path are all surfaces of standard parts, so only the correlations characteristics of them are analyzed without considering their geometric tolerance.

(6) Summary of geometric tolerance items

According to the above rules and specifications, the geometric tolerance items of each part in crankshaft piston mechanism can be obtained, as shown in Fig. 16.

(7) Summary of geometric tolerance items

According to the above rules and specifications, the geometric tolerance items of each part in crankshaft piston mechanism can be obtained, as shown in Fig. 16.
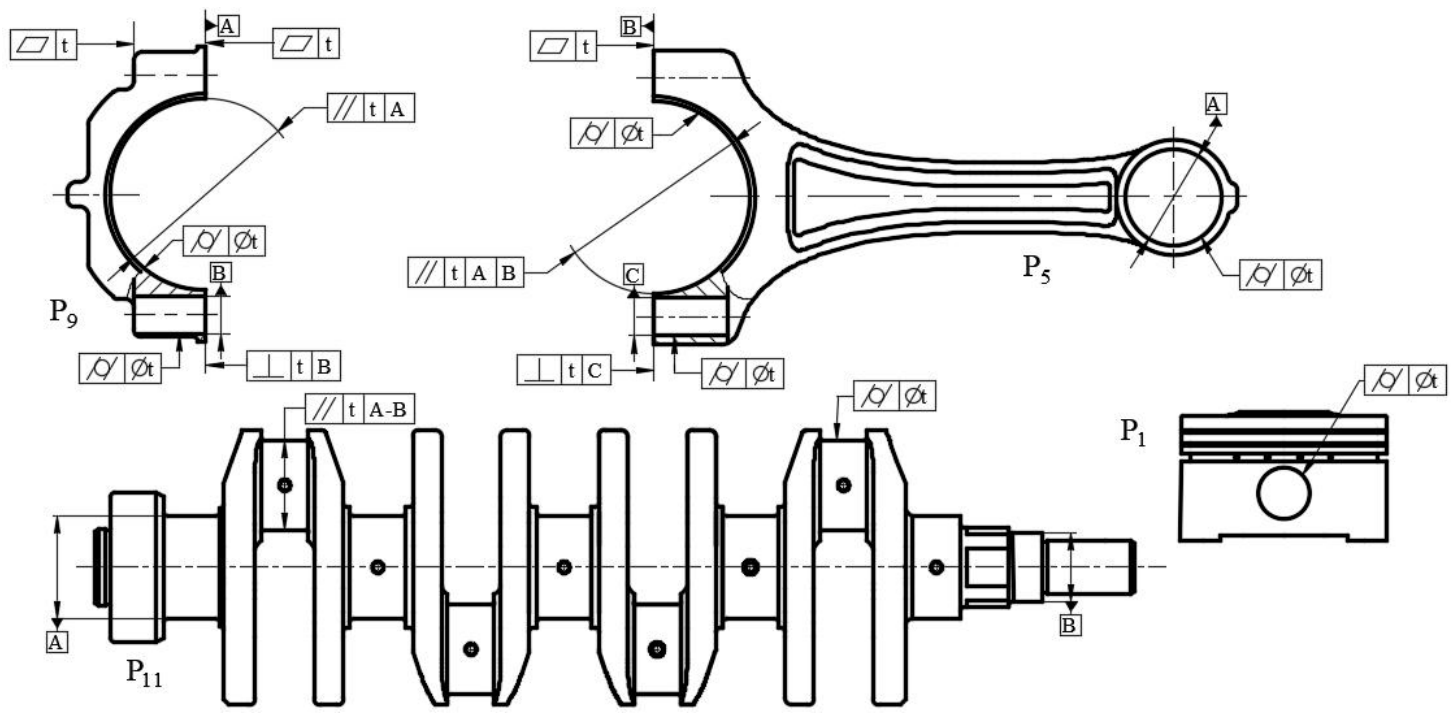

Fig. 16 Geometric tolerance items in crankshaft-piston mechanism

\section{Conclusion}

(1) Through the transferring method of geometric tolerance items based on assembly joints proposed in this paper, the top-down transfer process of geometric tolerance items at product-level, part-level and surface-level is defined, which is helpful to realize the synchronous design of geometric tolerance and product structure.

(2) The structural transformation of functional requirements is realized; The assembly joint was used as the basic structural unit of geometric tolerance item transmission; And the functional requirements and structural correlations between the correlated parts and the correlated surfaces were fully expressed by defining the contact type, mating surface type, constraint correlation type and the degree of freedom constraint. The rules of generating and transferring tolerance items based on assembly joints are established, which lays a foundation for selecting tolerance items.
(3) The transfer path of part-level based on functional requirements and structural correlations is established; On this basis, the matching constraints between the mating surfaces and the positioning constraints between the general structural surfaces are added. And the common mating surface is used as a link to sequentially connect with other mating surfaces that constitute assembly joints to form a continuous surface-level transfer path; On this basis, the functional requirements are transformed into the geometric tolerance items of the initial surface.

And along the path, the tolerance items between the same type of mating surfaces are copied, the tolerance items between different types of mating surfaces and between the general structural surfaces are converted. At the same time, the transfer of tolerance items is completed with the transfer of datums. The establishment of the transfer path improves the efficiency of tolerance design and is conducive to the automation and standardization of the geometric 
tolerance design process.

(4) Typical surfaces such as planes and cylinders are used as examples to carry out our research on the transfer method of geometric tolerance items based on assembly joints. Analysis of geometric tolerance items for complex surfaces are not yet involved. How to take the complex surface as the basic matching surface of the assembly joints and study the mechanism of tolerance transfer and transformation will be the content of further study. In addition, some atypical parts have the ambiguity of functional requirements analysis. How to accurately extract and analyze functional semantics and ensure the objectivity of structural transformation of functional requirements is also an important content to be studied in the future.

Acknowledgements The authors would like to acknowledge the fifinancial support by the National Natural Science Foundation of China (No. 51775239) and Natural Science Foundation Project of Shandong Province (ZR2020ME137).

Ethical Approval Not applicable.

Consent to Participate Not applicable.

Consent to Publish Not applicable.

Authors Contributions MA performed the design of method and wrote the manuscript; YANG and MA conceived and designed the study; LI, LIU, WANG, GAO helped perform the analysis with constructive discussions. All authors read and approved the manuscript.

Funding National Natural Science Foundation of China (No. 51775239) and Natural Science Foundation Project of Shandong Province (ZR2020ME137).

Competing Interests The authors have no conflicts of interest to declare that are relevant to the content of this article.

Availability of data and materials All data generated or analysed during this study are included in this published article.

\section{References}

1. Anselmetti B (2006). Generation of functional tolerancing based on positioning features. Comput Aided Des 38(8): 902-919.
2. Yang JX, Xu XS, Cao YL, et al. (2010) Functional tolerance specification design based on assembly positioning. CHIN J MECH ENG-EN 46(02): 1-8.

3. Gong JF, Ding LP, Chen BF, et al. (2016) Tolerance specification design for parts based on positioning features. AUTOMAT CONSTR 5: 16-21.

4. Zhang Y, Li ZB, Gao JM, et al. (2011) New reasoning algorithm for assembly tolerance specifications and corresponding tolerance zone types. Comput Aided Des 43(12): 1606-1628.

5. Armillotta A. (2013) A method for computer-aided specification of geometric tolerances. Comput Aided Des 45(12): 1604-1616.

6. Jiang K, Liu JH, Ning RX, et al. (2015) Geometric tolerances reasoning and its specification verification based on polychromatic sets theory. COMPUT INTEGR MANUF 21(03): 593-605.

7. Jaballi K, Bellacicco A, Louati J, et al. (2011) Rational method for 3D manufacturing tolerancing synthesis based on the TTRS approach "R3DMTSyn". COMPUT IND 62(5): 541-554.

8. Zhang K, Li Y, Tang S. (2010) An integrated modeling method of unified tolerance representation for mechanical product. INT J ADV MANUF TECH 46, 217 - 226.

9. Qin YC, Zhong YR, Chang L, et al. (2013) Automatic generation of tolerance types based on geometric tolerance description logic. COMPUT INTEGR MANUF 19(07): 1490- 1499.

10. Qin YC, Lu WL, Liu XJ, et al. (2015) Description logic-based automatic generation of geometric tolerance zones. INT J ADV MANUF TECH 79(5-8): 1221-1237. 11. Qin YC, Lu WL, Qi QF, et al. (2018) Towards a tolerance representation model for generating tolerance specification schemes and corresponding tolerance zones. INT J ADV MANUF TECH 97: 1801-1821.

12. Zhong YR, Qin YC, Huang MF, et al. (2013) Automatically generating assembly tolerance types with an ontology-based approach. Comput Aided Des 45(11): $1253-1275$

13. Zhong YR, Qin YC, Huang MF, et al. (2014) Constructing a meta-model for assembly tolerance types with a description logic based approach. Comput Aided Des 48: 1-16. 
14. Zhao Q, Li T, Cao Y, et al. (2020) A computer-aided tolerance specification method based on multiple attributes decision-making. INT J ADV MANUF TECH 111, 1735 - 1750.

15. Sarigecili M I, Roy U, Rachuri S. (2014) Interpreting the semantics of GD\&T specifications of a product for tolerance analysis. Comput Aided Des 47:72-84.
16. Goetza S, and Schleicha B. (2020) Ontology-based representation of tolerancing and design knowledge for an automated tolerance specification of product concepts. Procedia CIRP 92: 194-199.

17. Wang HQ, Lv ZY, Zhang X, et al. (2018) GD \& T semantic information interpretation for tolerance analysis. COMPUT INTEGR MANUF 24(04): 994-1006. 
Figures

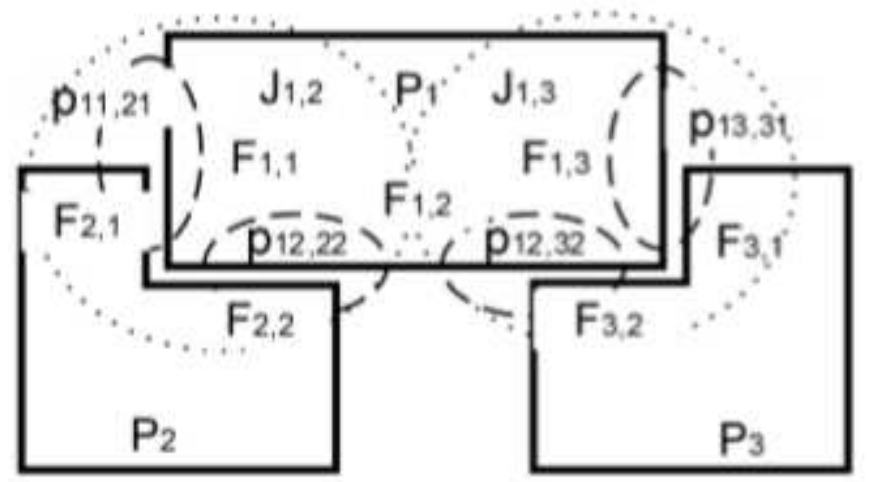

Figure 1

The relationship between assembly joint and mating surface pairs

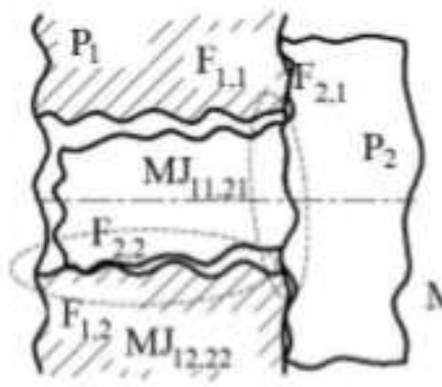

(a)

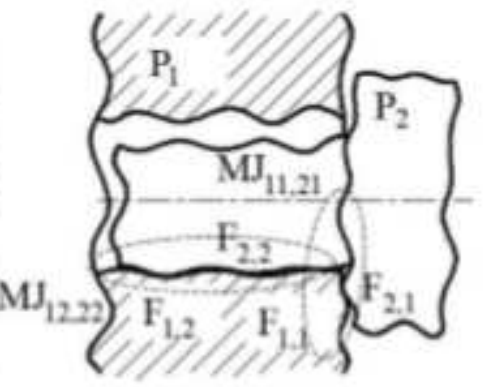

(b)

Figure 2

Schematic diagram 1 of the priority of assembly joints

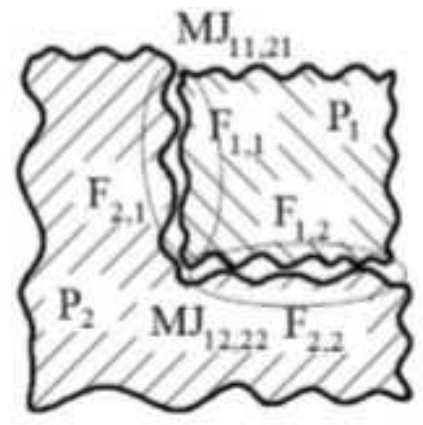

(a)

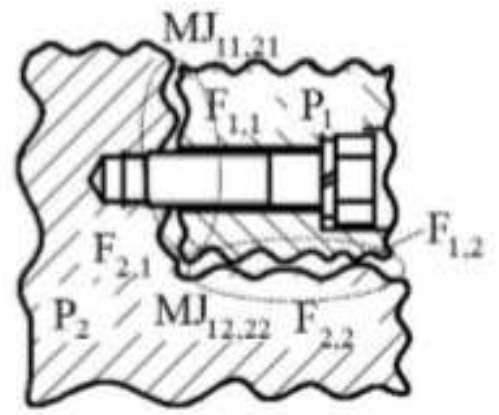

(b)

Figure 3

Schematic diagram 2 of the priority of assembly joints 

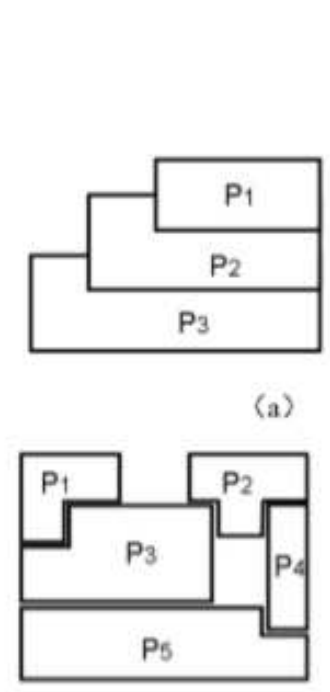

(c)
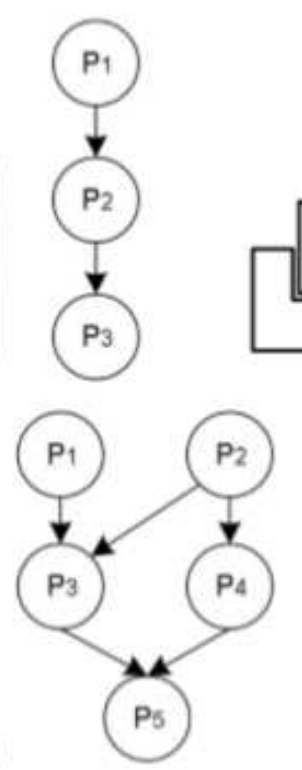

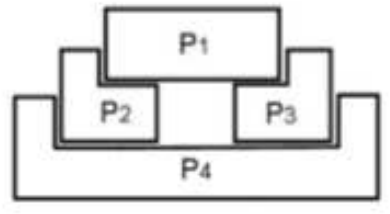

(b)

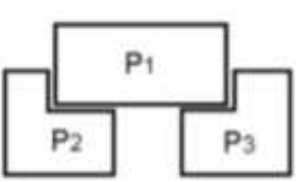

(d)
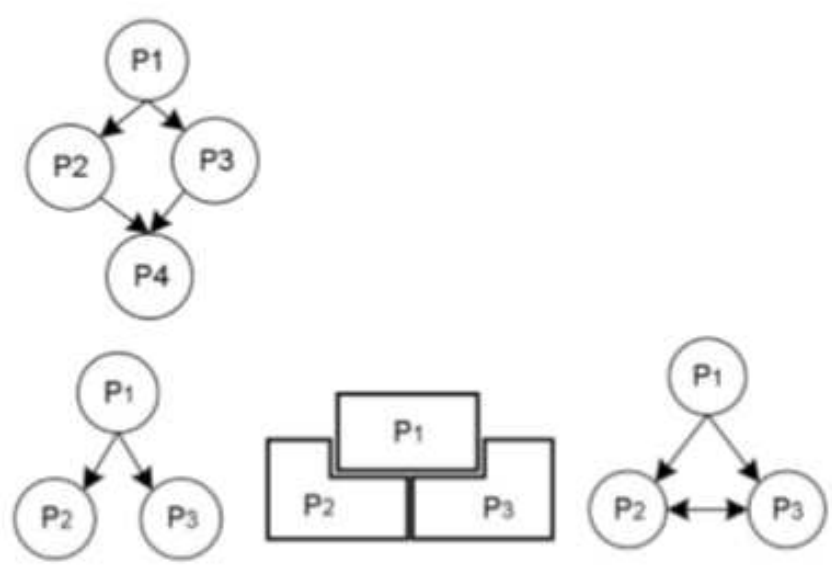

(e)

Figure 4

Basic form of the transfer path of part-level

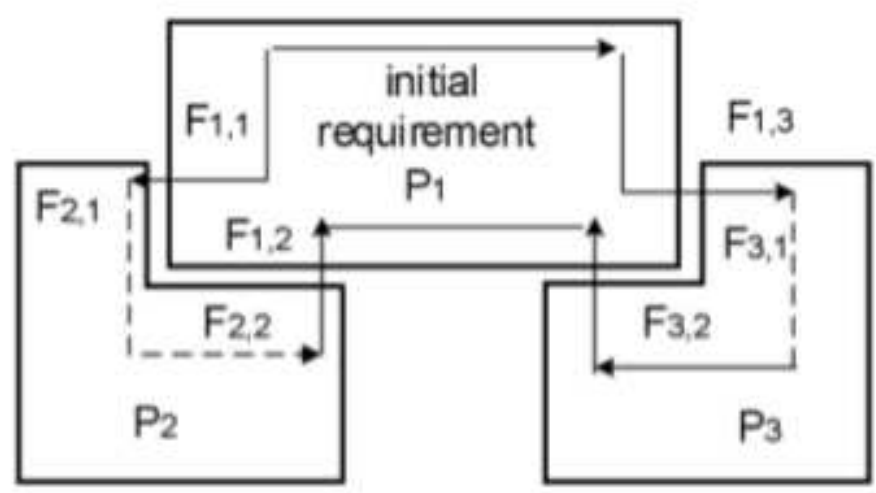

(a)

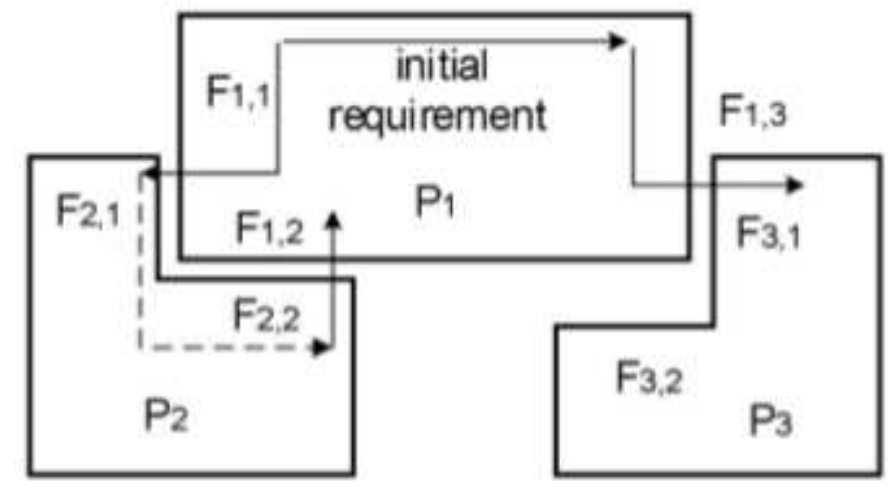

(b)

Figure 5

Transfer path of surface-level 


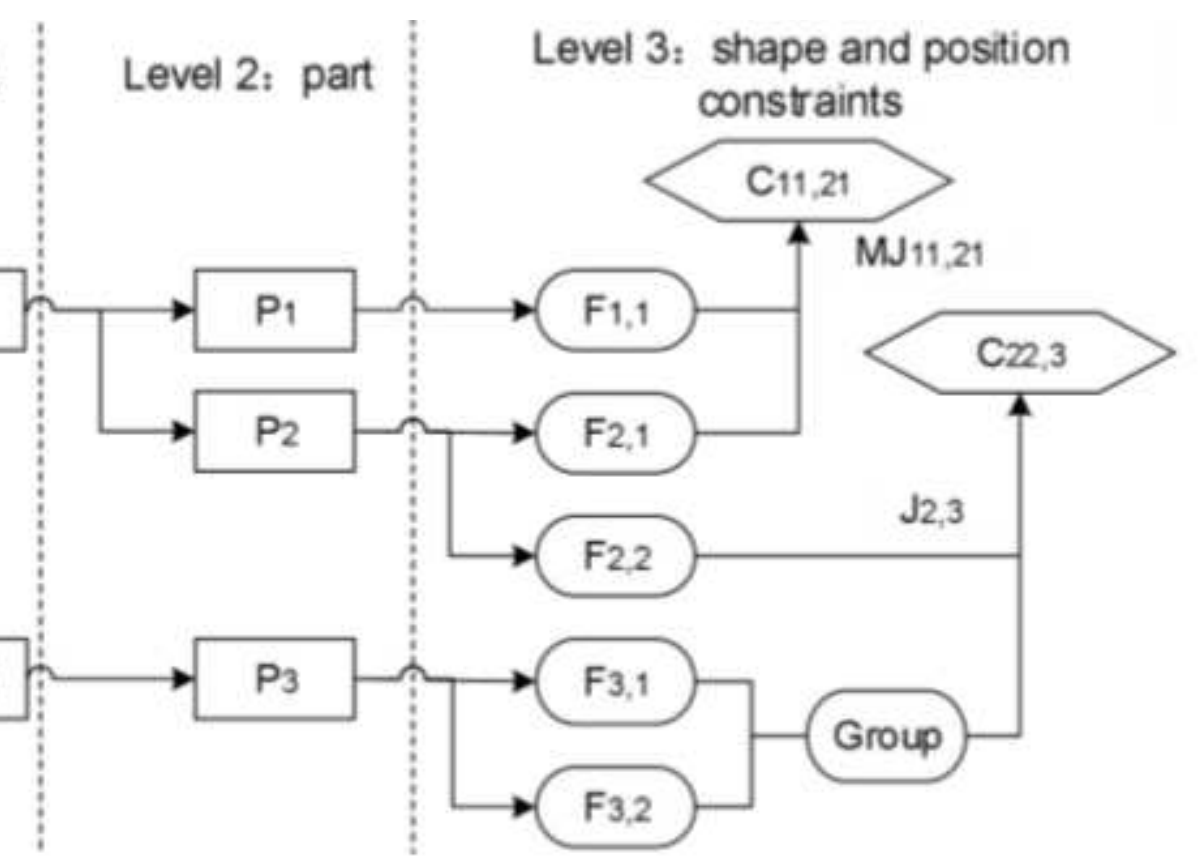

Figure 6

Transformation Model of functional requirements

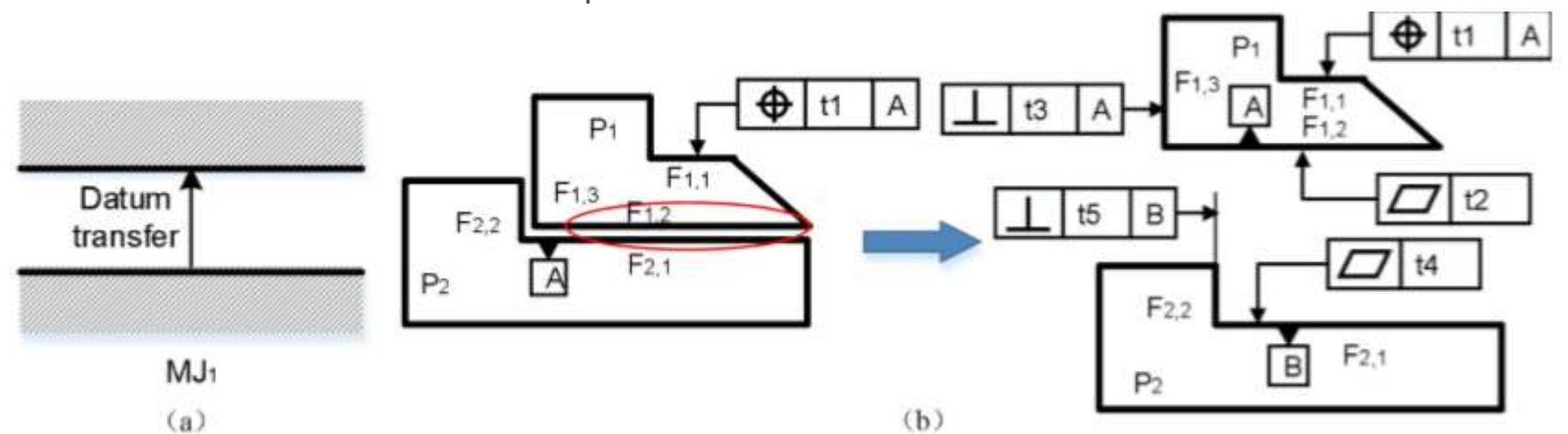

Figure 7

Datum transfer based on assembly joint 1

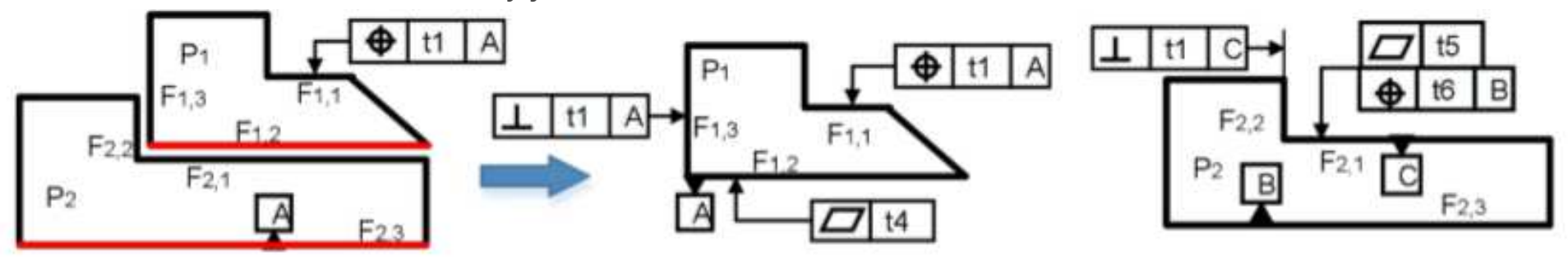

Figure 8

Datum transfer based on assembly joint 2 

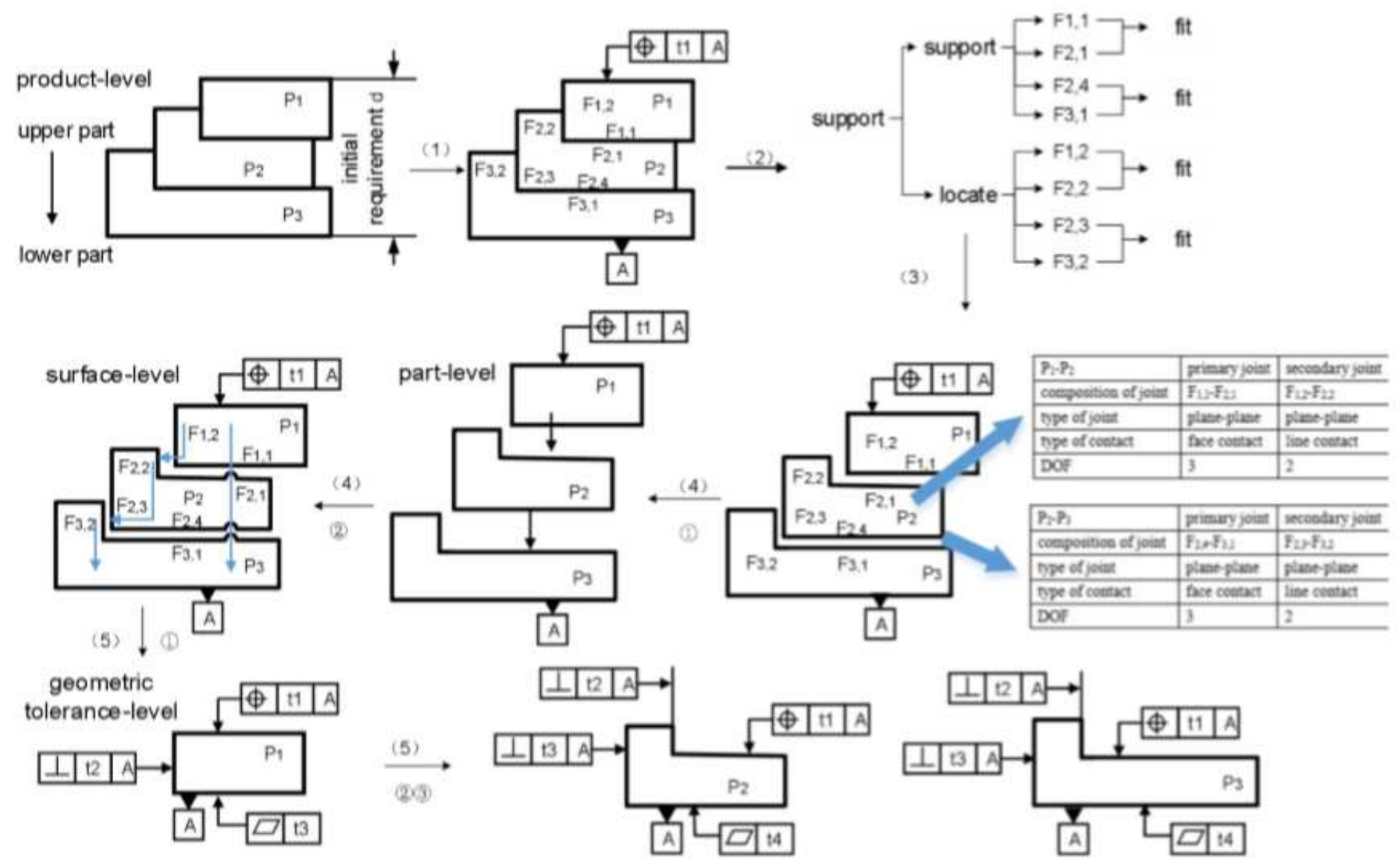

\section{Figure 9}

Transfer process of geometric tolerance items based on assembly joints

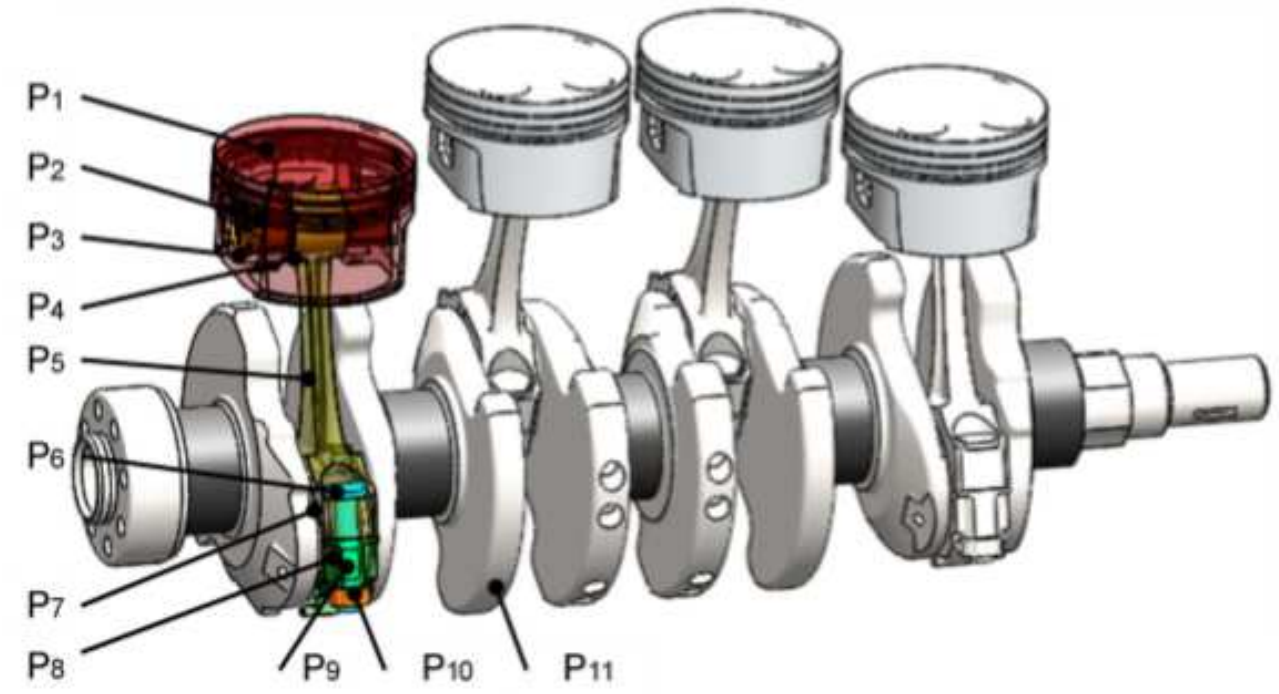

$\mathrm{P}_{1}$-piston, $\mathrm{P}_{2}$-piston pin, $\mathrm{P}_{3}$-retaining ring, $\mathrm{P}_{4}$-rod bushing, $\mathrm{P}_{5}$-connecting rod, $\mathrm{P}_{6}$-bolt, $\mathrm{P}_{7}$-bearing bush 1 , $\mathrm{P}_{8}$-bearing bush 2, $\mathrm{P}_{9}$-connecting rod cover, $\mathrm{P}_{10}$-nut, $\mathrm{P}_{11}$-crankshaft

\section{Figure 10}

Crankshaft piston mechanism of engine 


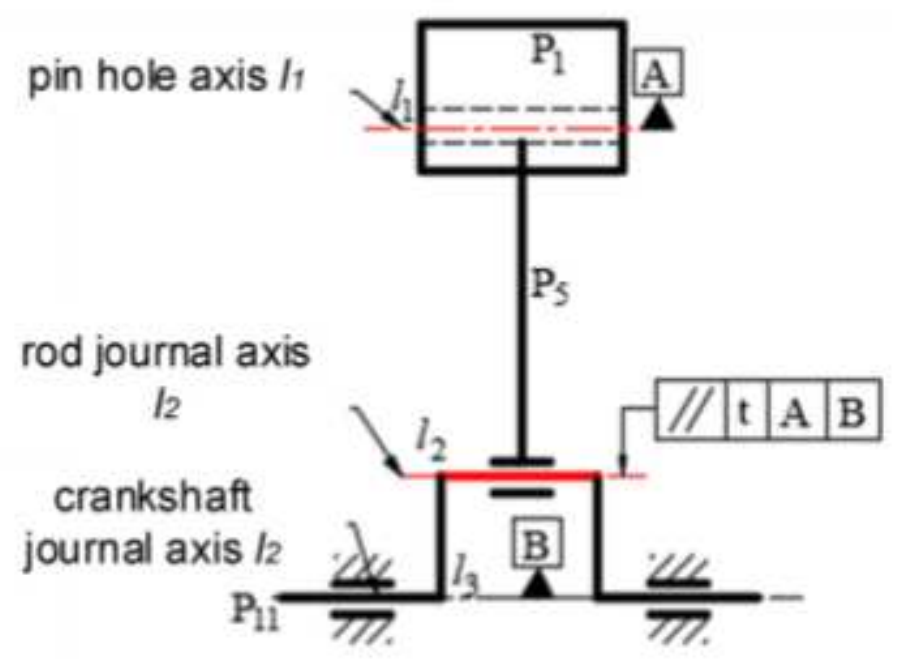

Figure 11

Transformation of initial design requirements to geometric tolerance items

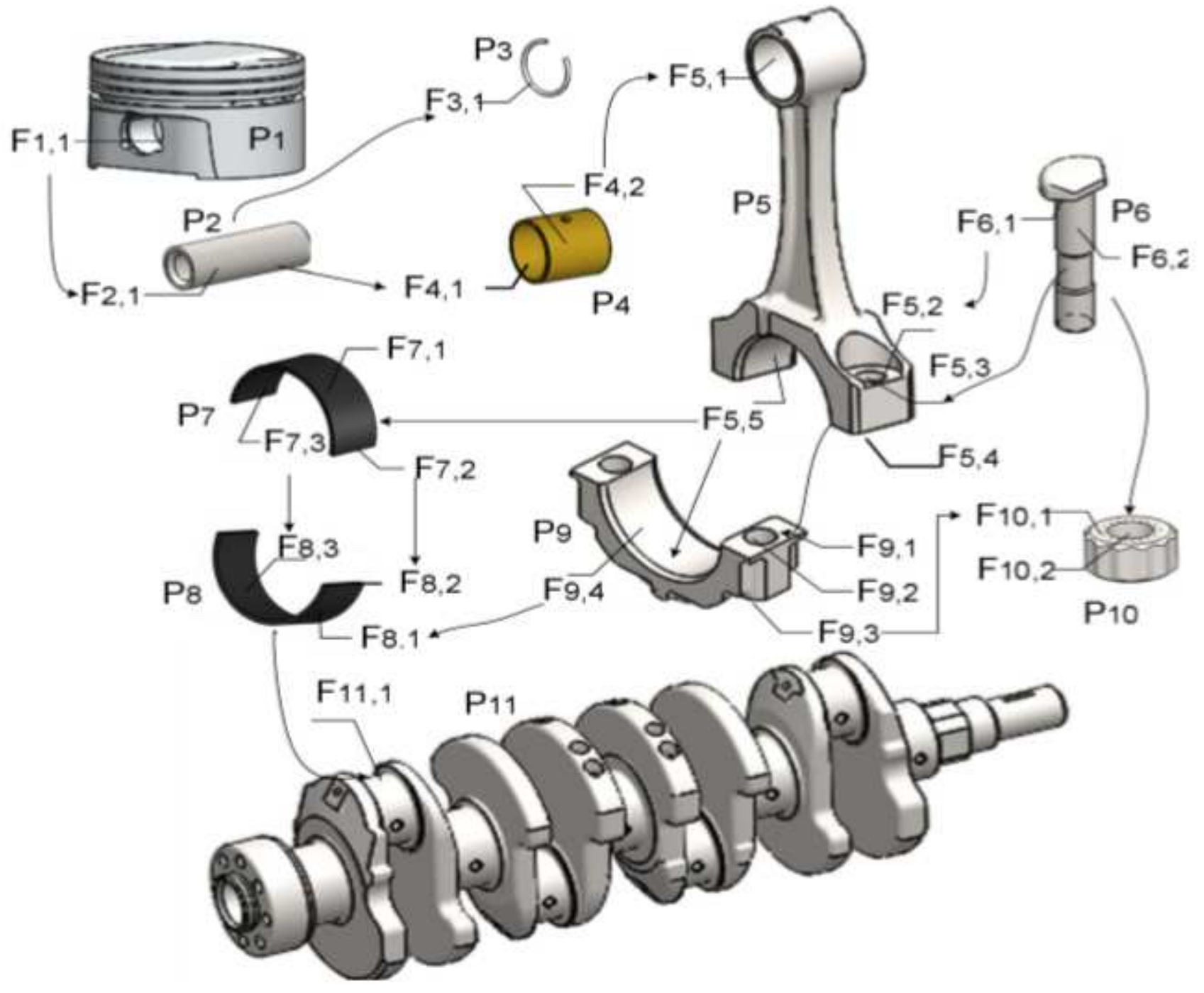

Figure 12 
The functional surface of the crank shaft-piston mechanism and the correlation model

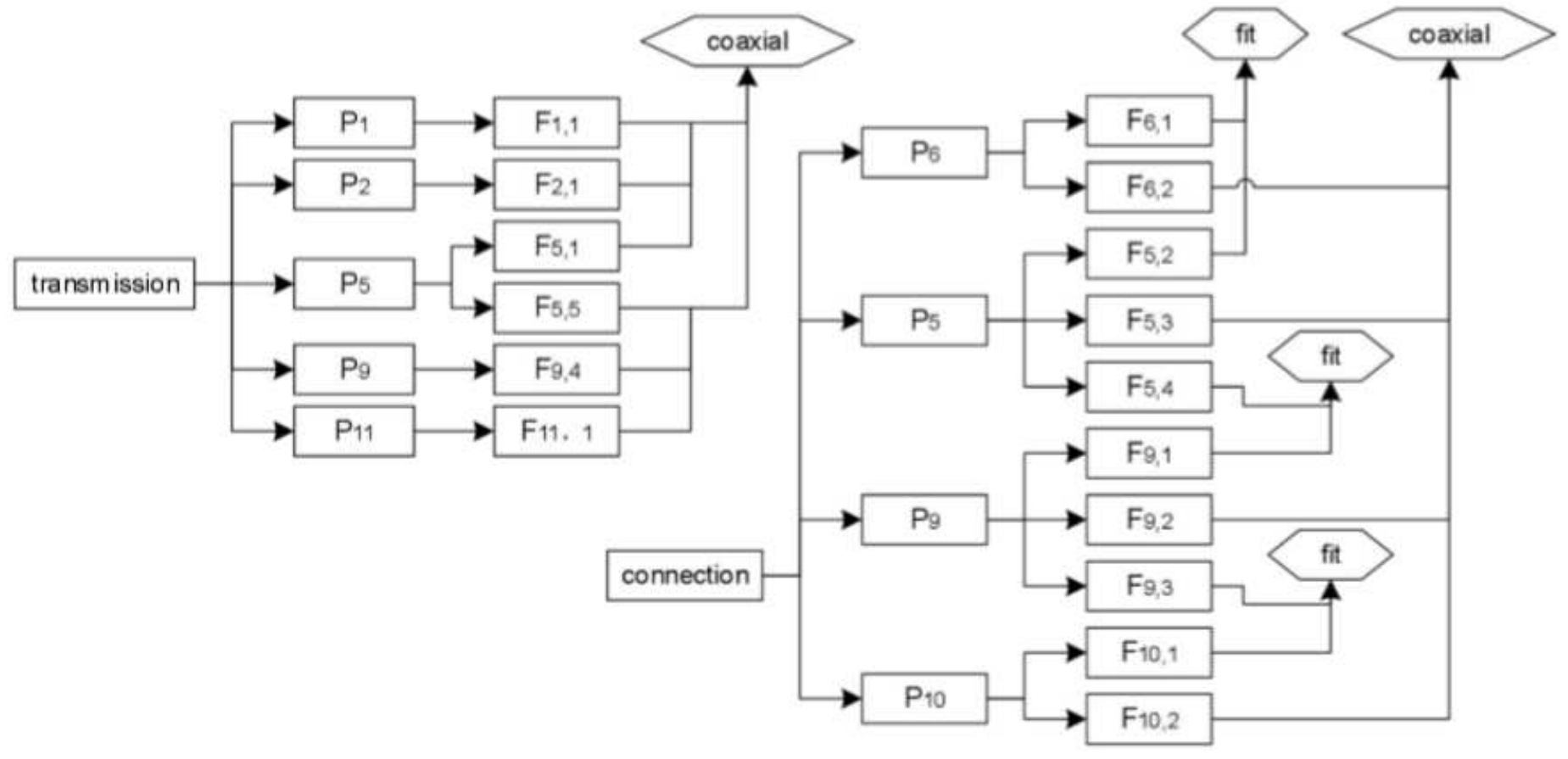

Figure 13

Structural transformation model of functional requirement

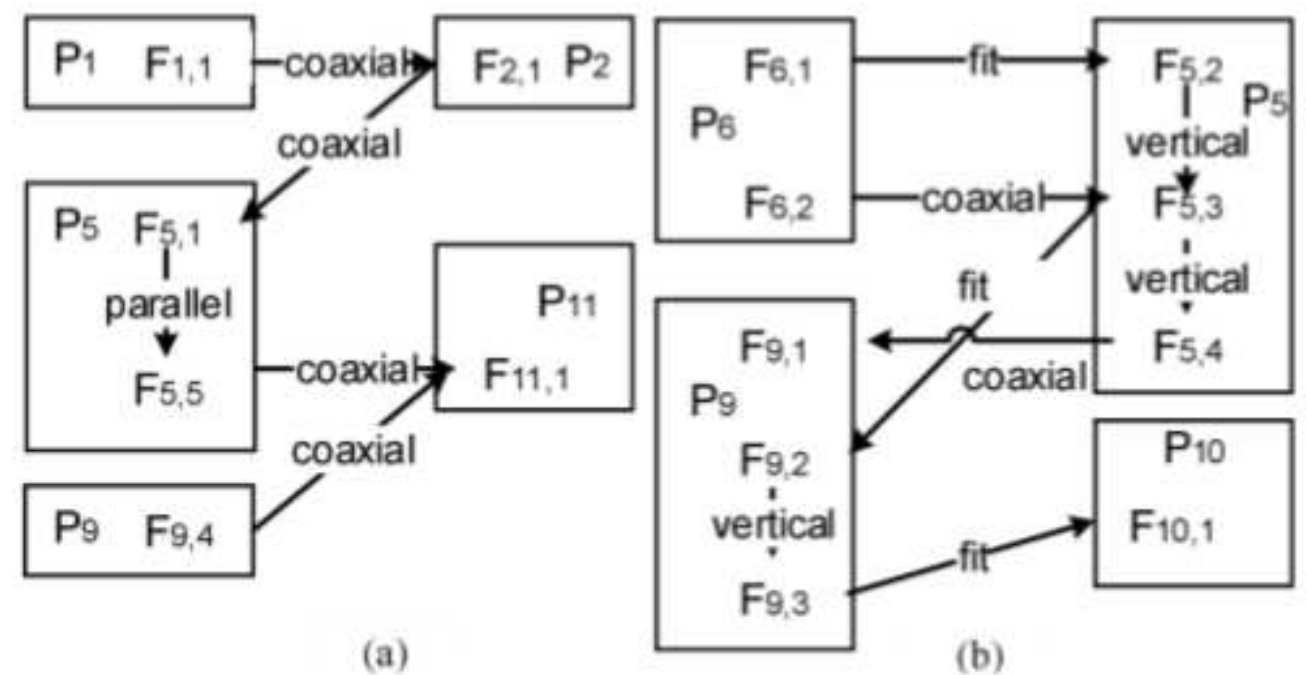

Figure 14

Graphical representation of the surface-level transfer paths 
(2) Transfer of datum

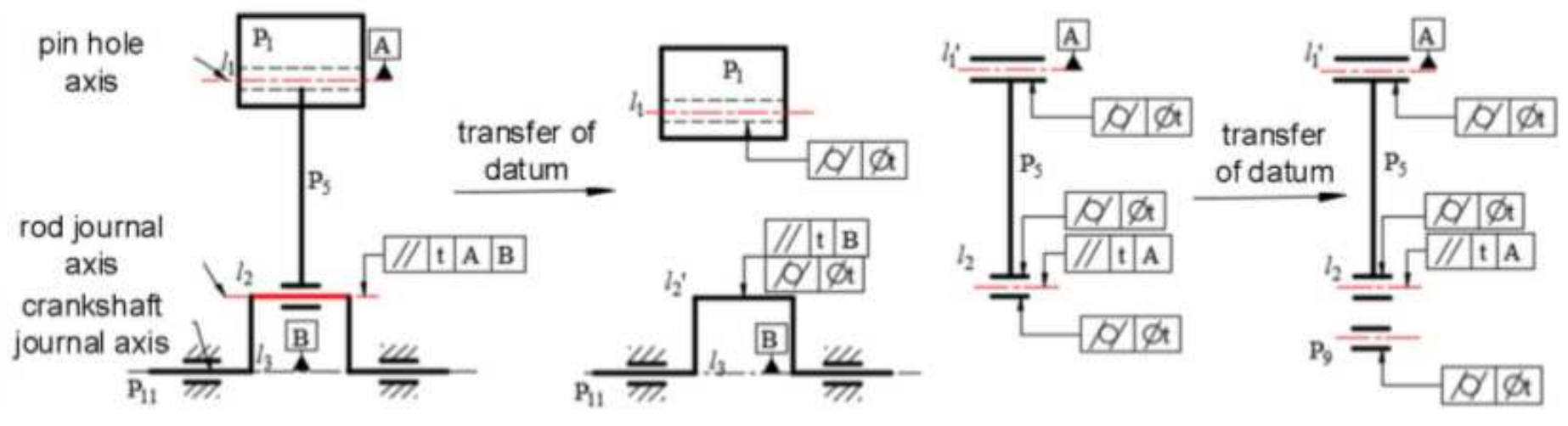

Figure 15

Transfer of datums under the initial design requirement

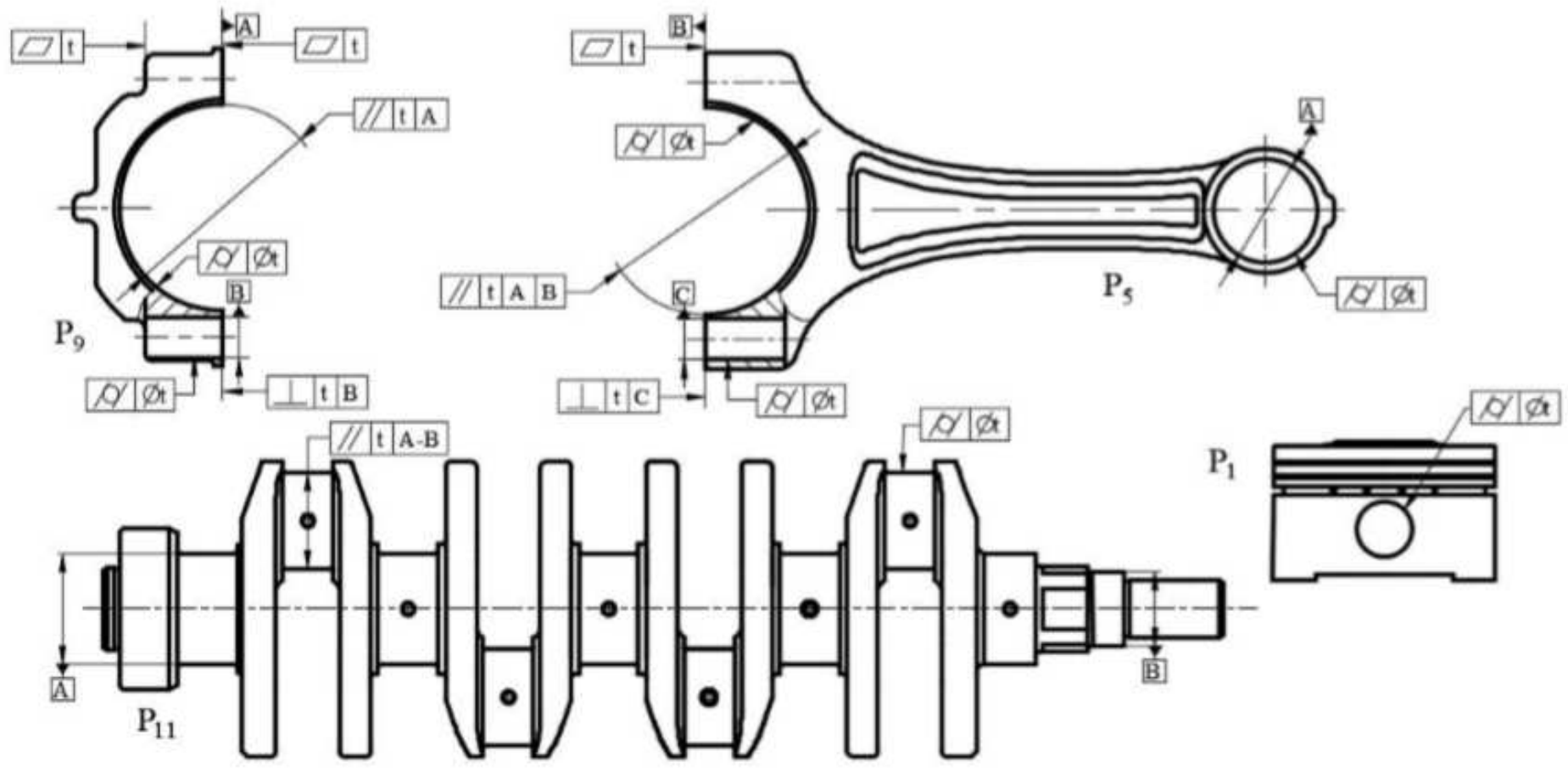

Figure 16

Geometric tolerance items in crankshaft-piston mechanism 